\title{
Transport Coefficients of Hyperonic Neutron Star Cores
}

\author{
Peter Shternin ${ }^{1, *(D)}$ and Isaac Vidaña ${ }^{2}$ D \\ 1 Ioffe Institute, 26 Politekhnicheskaya St., 194021 St. Petersburg, Russia \\ 2 INFN Sezione di Catania, Via Santa Sofia 64, 95123 Catania, Italy; isaac.vidana@ct.infn.it \\ * Correspondence: pshternin@gmail.com
}

\section{check for}

updates

Citation: Shternin, P.; Vidaña, I. Transport Coefficients of Hyperonic Neutron Star Cores. Universe 2021, 7, 203. https://doi.org/10.3390/ universe7060203

Academic Editor: Fridolin Weber

Received: 22 April 2021

Accepted: 15 June 2021

Published: 20 June 2021

Publisher's Note: MDPI stays neutral with regard to jurisdictional claims in published maps and institutional affiliations.

Copyright: (c) 2021 by the authors. Licensee MDPI, Basel, Switzerland. This article is an open access article distributed under the terms and conditions of the Creative Commons Attribution (CC BY) license (https:// creativecommons.org/licenses/by/ $4.0 /)$.

\begin{abstract}
We consider transport properties of the hypernuclear matter in neutron star cores. In particular, we calculate the thermal conductivity, the shear viscosity, and the momentum transfer rates for $\mathrm{np} \Sigma^{-} \Lambda e \mu$ composition of dense matter in $\beta$-equilibrium for baryon number densities in the range $0.1-1 \mathrm{fm}^{-3}$. The calculations are based on baryon interactions treated within the framework of the non-relativistic Brueckner-Hartree-Fock theory. Bare nucleon-nucleon (NN) interactions are described by the Argonne v18 phenomenological potential supplemented with the Urbana IX three-nucleon force. Nucleon-hyperon (NY) and hyperon-hyperon (YY) interactions are based on the NSC97e and NSC97a models of the Nijmegen group. We find that the baryon contribution to transport coefficients is dominated by the neutron one as in the case of neutron star cores containing only nucleons. In particular, we find that neutrons dominate the total thermal conductivity over the whole range of densities explored and that, due to the onset of $\Sigma^{-}$which leads to the deleptonization of the neutron star core, they dominate also the shear viscosity in the high density region, in contrast with the pure nucleonic case where the lepton contribution is always the dominant one.
\end{abstract}

Keywords: neutron stars; transport properties; hypernuclear matter

\section{Introduction}

The interior composition of neutron stars (NSs) —among the most dense material objects in the Universe-is not known yet and various phases of dense matter might be expected in their interiors, see, e.g., Ref. [1], for a recent review. With masses of the order of the Solar mass and $\sim 10 \mathrm{~km}$ radii, the density of matter in the innermost regions of NSs, their cores, can reach values a few times that of symmetric nuclear matter at saturation, $n_{0}=0.16 \mathrm{fm}^{-3}$. In the simplest model, the matter of NS cores consists of uniform fluid of neutrons $(n)$, protons $(p)$, and leptons (electrons $(e)$ and muons $(\mu)$ ) in equilibrium with respect to the weak interaction ( $\beta$-stable matter). However, at densities of about (2-3) $n_{0}$ simple energy arguments suggest that other baryonic degrees of freedom, such as, for instance, hyperons, can appear. This possibility was first proposed by Ambartsumyan and Saakyan [2] in 1960 and it has been later extensively studied in great detail by many authors using different phenomenological [3-12] or microscopical [13-26] approaches to the equation of state (EOS) of NS matter with hyperons. However, although the presence of hyperons in NSs seems to be energetically unavoidable, the softening that their presence induces on the EOS leads to NS maximum masses which are incompatible with the observation of the unusually high masses of the millisecond pulsars PSR J1903 + $0327\left(1.667 \pm 0.021 M_{\odot}\right)$ [27], PSR J1614-2230 $\left(1.928 \pm 0.017 M_{\odot}\right)$ [28], PSR J0348 + $0432\left(2.01 \pm 0.04 M_{\odot}\right)$ [29] and the most recent one PSR J0740+6620 $\left(2.14_{-0.09}^{+0.10} M_{\odot}\right)$ [30]. The solution of this problem, commonly known in the literature as the 'hyperon puzzle', is not easy and it has become a subject of very active research in recent years, see, e.g., Refs. [31,32] and references therein for a recent and comprehensive review. An alternative way to circumvent the hyperon puzzle is to invoke the appearance of other hadronic degrees of freedom that push the onset of hyperons to higher densities, such as for instance meson condensates. The possible existence of a Bose-Einstein condensate of negative kaons in the inner core of NSs has been 
also extensively considered in the literature (see e.g., Refs. [33-37] and references therein). When the $K^{-}$chemical potential, $\mu_{K^{-}}$, becomes smaller than the one of the electron, the process $e^{-} \rightarrow K^{-}+v_{e}$ becomes energetically possible and kaons condensate in the NS interior. The onset density for this process was calculated to be in the range $(2.5-5) n_{0}[35,36]$. However, the appearance of kaon condensation induces also a strong softening of the EOS and consequently leads to the reduction of the NS maximum mass, as in the case of hyperons, to values below the current observational limits. The role of kaons in NSs is out of the scope of the present paper and the interested reader is referred to the original works on this topic [33-37] for a comprehensive description of the implications of kaon condensation on the structure and evolution of NSs, and to Ref. [38] for a recent review of the present status of experimental and theoretical developments on kaon physics.

Neutron stars are evolving entities, where various dynamical processes are believed to occur. One can mention among others, for instance, NS cooling, NS oscillations due to undamped instabilities in rotating stars, or magnetic field dissipation. The theoretical modelling of these processes requires knowledge of the transport properties of dense NS matter [39]. In recent studies, the transport properties of ultradense nucleon or pure quark matter have received considerable attention, see e.g., Ref. [39] for a review. Conversely, the coverage of the strange sector has been much lower. Therefore, in this paper we make a step in the direction of filling this gap and consider transport coefficients of the hypernuclear NS core matter. Specifically, we focus on the thermal conductivity and shear viscosity as well as on the momentum transfer rates in binary collisions. The latter coefficients describe friction between the components of the matter in the diffusion process. If the diffusion is caused by the external electromagnetic field, these coefficients enter the generalized Ohm law and determine the electrical conductivity, which is important for studies of the magnetic field evolution of NSs, see, e.g., Refs. [39-42]. Our results are based on the classical formalism of the kinetic theory of Fermi systems [43] adapted for the NS context, see Refs. [39,44-47], and references therein.

The basic problem in the transport theory of strongly interacting systems lies in the poor knowledge of many-body interactions at high densities. Various methods are employed, often having poor control of the underlying assumptions and approximations in the high density region. Even within the same theoretical framework, differences in the microphysical input may lead to drastically different results. For instance, for nuclear matter it was found that various nuclear potential models, all behaving well at low densities, result in an order of magnitude difference of the transport properties at several nuclear densities [47]. In the case of hypernuclear matter the situation is even more tricky, because the nucleon-hyperon (NY) and hyperon-hyperon (YY) interactions are, unfortunately, still poorly constrained. Contrary to the nucleon-nucleon (NN) interaction, which is fairly well known due to the larger number of existing scattering data and measured properties of nuclei, experimental data in the hyperonic sector (see Refs. [31,32,38] and references therein) is not sufficient yet to constrain these interactions with a comparable precision.

In the present work we calculate the transport coefficients of $\mathrm{np} \Sigma^{-} \Lambda e \mu$ matter. The number fractions of different matter constituents are found from the requirements of electric charge neutrality and $\beta$-stability. The EOS of the hyperonic matter is obtained within the non-relativistic Brueckner-Hartree-Fock (BHF) approach using realistic NN, NY, and YY interactions, see Ref. [48] for a general overview of this approach and Refs. [13-18] for details of its application to hypernuclear matter. As a first step, in this work, we use a couple of interaction models for the NY and YY interactions in order to explore the difference with the results for pure nucleonic compositions; a more systematic study of the dependence of the results on the choice of baryon interaction is left for future works. Within the nucleonic sector we use, in particular, the Argonne v18 (Av18 for short) potential [49] supplemented with an effective density-dependent two-body force derived from the three-nucleon force of the Urbana IX (UIX for short) model [50]. For the interactions involving hyperons we employ two versions of the Nijmegen Soft-Core 97 (NSC97 in the next) meson-exchange model [51,52], namely, the models NSC97e and NSC97a. Three-body interactions involving 
hyperons are, unfortunately, still quite uncertain and, therefore, are not included in this work. In the following, since the nucleonic part is described always with the Av18 NN potential plus the UIX NNN force, for simplicity we will use the labels NSC97e and NSC97a when referring to the two sets of models used.

The paper is organized as follows. In Section 2 we briefly review the basic expressions for the transport coefficients of Fermi systems. In Section 3 we shortly present the BHF approach of hypernuclear matter. We present the transport coefficients in Section 4 , and conclude in Section 5. In what follows, we set $\hbar=k_{B}=c=1$.

\section{Transport Coefficients}

Let us briefly review the formalism used to calculate the transport coefficients in the case of neutron star cores with hyperons. A detailed description of this formalism for the nuclear matter case can be found in Refs. [46,47,53].

The basic expressions for calculating the thermal conductivity $\kappa_{c}$ and the shear viscosity $\eta_{c}$ of particle species $c$, and the momentum transfer rates $J_{c i}$ in the binary collisions between particle species $c$ and $i$ are

$$
\begin{aligned}
\kappa_{c} & =\frac{\pi^{2}}{3} T \frac{n_{c}}{p_{F c}} \lambda_{c}^{\kappa}, \\
\eta_{c} & =\frac{1}{5} n_{c} p_{F c} \lambda_{c}^{\eta}, \\
J_{c i} & =n_{c} p_{F c}\left(\lambda_{c i}^{D}\right)^{-1},
\end{aligned}
$$

where $T$ is the temperature, $n_{c}$ and $p_{F c}$ are, respectively, the particle number densities and Fermi momenta, $\lambda_{c}^{\alpha}$ with $\alpha=\kappa, \eta$, and $\lambda_{c i}^{D}$ are the effective mean free paths which are, in general, different for the different transport coefficient in question. Having this in mind, below we drop the index $\alpha$ for brevity and reinstall it only when necessary. The effective mean free paths are obtained in the kinetic theory from the solution of the system of linearized transport equations for the distribution functions of the quasiparticles.

The simplest approximation, which however was proved to be very good in degenerate matter, is the lowest-order variational approximation, in which case the system of transport equations reduces to the system of linear equations for the effective mean free paths

$$
\sum_{i} \Lambda_{c i} \lambda_{i}=1
$$

The matrix of this system is the transport matrix $\Lambda_{c i}$, which is connected to the effective transport cross-sections $\sigma_{c i}, \sigma_{c i}^{\prime}$ of binary collisions as

$$
\begin{aligned}
\Lambda_{c c} & =\sum_{i} n_{i} \sigma_{c i}+n_{c} \sigma_{c c^{\prime}}^{\prime} \\
\Lambda_{c i} & =n_{i} \sigma_{c i}^{\prime}, \quad i \neq c .
\end{aligned}
$$

Effective transport cross-sections $\sigma_{c i}$ and $\sigma_{c i}^{\prime}$ in Equations (5) and (6) are obtained by averaging of a squared matrix element of the scattering matrix with certain angular factors. In our case of $\mathrm{np} \Sigma^{-} \Lambda e \mu$ matter, particles interact via the electromagnetic and strong forces. Leptons participate only in the electromagnetic interactions, while the strong interaction is the dominant channel for the baryons (see, however, Section 4.1). The explicit integral expressions for the transport cross-sections convenient to use for the strong interactions can be found in Ref. [47] and the form of these quantities more convenient for electromagnetic interactions is given, e.g., in Ref. [54]. 
The momentum transfer rates in Equation (3) are usually treated in the lowest-order moment expansion of the kinetic theory which is practically equivalent to the variational method described above. The effective partial mean free paths for binary collisions are

$$
\left(\lambda_{c i}^{D}\right)^{-1}=n_{i} \sigma_{c i}^{D}
$$

where $\sigma_{c i}^{D}$ is the transport cross-section for the diffusion problem [47].

Notice, that the transport matrices in Equations (4)-(6) are not symmetric. Nondiagonal matrix elements of the transport matrices obey the relations $n_{c} p_{F c}^{-1} \Lambda_{c i}^{\kappa}=n_{i} p_{F i}^{-1} \Lambda_{i c}^{\kappa}$ and $n_{c} p_{F c} \Lambda_{c i}^{\eta}=n_{i} p_{F i} \Lambda_{i c}^{\eta}$ [47]. The momentum transfer rates in Equation (3) are symmetric, $J_{c i}=J_{i c}$.

In the quasiparticle approximation, only the collisions in the vicinity of the Fermi surface contribute to transport. If the matrix element does not depend on the energy transfer in collisions (which is $\sim T$ ), one finds the simple scaling rules valid for Fermi systems at low temperatures, $\sigma_{c i} \propto m_{c}^{* 2} m_{i}^{* 2} T^{2}$, where $m_{c}^{*}$ and $m_{i}^{*}$ are the quasiparticles effective masses on the Fermi surface [43]. This is the case for collisions mediated by the nuclear forces. Transport cross-sections governed by the electromagnetic interactions in NS cores obey non-Fermi liquid temperature dependence as a result of the dynamical character of the plasma screening in the dominant transverse channel of the electromagnetic interaction $[55,56]$. The matrix elements of the 'electromagnetic' transport matrix do not follow $\propto T^{2}$ scaling, but, generally, have weaker temperature dependence; the exact scaling is subject to the transport problem in question [39].

\section{Brueckner-Hartree-Fock Approach of Hypernuclear Matter}

Calculations of the transport cross-sections, and consequently of the transport coefficients, require as an input the squares of the different scattering transition matrix elements and the effective masses of the involved baryons which should be provided by a nuclear many-body theory. Various methods have been considered to solve the nuclear many-body problem: the variational approach [57], the correlated basis function (CBF) formalism [58], the self-consistent Green's function (SCGF) technique [59,60], or the Brueckner-Bethe-Goldstone (BBG) [61] and the Dirac-Bruecker-Hartree-Fock (DBHF) theories [62-64]. Nevertheless, although all of them have been extensively applied to the study of nuclear matter, up to our knowledge, only the BBG theory in the BHF approximation [13-19,22,25,26], and very recently the DBHF theory [20], the V-low-k approach [21], and the quantum Monte Carlo method [23,24] have been extended to the hyperonic sector.

In this section we briefly review the non-relativistic Brueckner-Hartree-Fock approximation of hypernuclear matter. We would like to note that although this approach is non-relativistic we found that the speed of sound is, in all the range of densities considered here, always smaller than the speed of light. The reason is that the softening of the EOS induced by the appeareance of the hyperons guarantees causality. The BHF approximation starts with the construction of all baryon-baryon (NN, YN, and YY) G-matrices which describe the interaction between two baryons in the presence of a surrounding medium. The G-matrices are obtained by solving the well-known coupled-channel Bethe-Goldstone integral equation

$$
\begin{gathered}
\left\langle\vec{k}_{B_{1}} \vec{k}_{B_{2}}|G(\omega)| \vec{k}_{B_{3}} \vec{k}_{B_{4}}\right\rangle=\left\langle\vec{k}_{B_{1}} \vec{k}_{B_{2}}|V| \vec{k}_{B_{3}} \vec{k}_{B_{4}}\right\rangle \\
+\sum_{B_{i} B_{j}} \frac{\left\langle\vec{k}_{B_{1}} \vec{k}_{B_{2}}|V| \vec{k}_{B_{i}} \vec{k}_{B_{j}}\right\rangle\left\langle\vec{k}_{B_{i}} \vec{k}_{B_{j}}|Q| \vec{k}_{B_{i}} \vec{k}_{B_{j}}\right\rangle\left\langle\vec{k}_{B_{i}} \vec{k}_{B_{j}}|G(\omega)| \vec{k}_{B_{3}} \vec{k}_{B_{4}}\right\rangle}{\omega-E_{B_{i}}\left(k_{B_{i}}\right)-E_{B_{j}}\left(k_{B_{j}}\right)+i \eta},
\end{gathered}
$$

where $V$ is the bare baryon-baryon interaction, $Q$ is the Pauli operator that prevents the intermediate baryons $B_{i}$ and $B_{j}$ from being scattered to states below their respective Fermi 
momenta, $\omega$ is the sum of the non-relativistic single-particle energies of the interacting baryons, and $\eta$ is an infinitesimal positive quantity.

The single-particle energy of a baryon $B_{i}$ is given by

$$
E_{B_{i}}\left(k_{B_{i}}\right)=m_{B_{i}}+\frac{k_{B_{i}}^{2}}{2 m_{B_{i}}}+\operatorname{Re}\left[U_{B_{i}}\left(k_{B_{i}}\right)\right],
$$

where $m_{B_{i}}$ denotes the rest mass of the baryon, and the (complex) single-particle potential $U_{B_{i}}\left(k_{B_{i}}\right)$ represents the average field 'felt' by the baryon owing to its interaction with other baryons. In the BHF approximation, $U_{B_{i}}\left(k_{B_{i}}\right)$ is calculated through the 'on-shell' G-matrix, and is given by

$$
U_{B_{i}}\left(k_{B_{i}}\right)=\sum_{B_{j}} \sum_{\vec{k}_{B_{j}}} n_{B_{j}}\left(k_{B_{j}}\right)\left\langle\vec{k}_{B_{i}} \vec{k}_{B_{j}}\left|G\left(\omega=E_{B_{i}}\left(\vec{k}_{B_{i}}\right)+E_{B_{j}}\left(\vec{k}_{B_{j}}\right)\right)\right| \vec{k}_{B_{i}} \vec{k}_{B_{j}}\right\rangle_{\mathcal{A}} .
$$

Here $n_{B_{j}}\left(k_{B_{j}}\right)$ is the occupation number of the baryon species $B_{j}$, and the index $\mathcal{A}$ indicates that the matrix elements are properly antisymmetrized when baryons $B_{i}$ and $B_{j}$ belong to the same isomultiplet. We note here that the so-called continuous prescription has been adopted for the single-particle potentials when solving the Bethe-Goldstone equation, since, as it was shown in Refs. $[65,66]$, the contribution to the energy per particle from threehole line diagrams is minimized in this prescription. As we already said in the introduction all calculations have been carried out with the Av18 NN potential [49] supplemented with the UIX three-nucleon force [50], which, for the use in the BHF calculations, was reduced to a two-body density-dependent force by averaging over the spatial, spin, and isospin coordinates of the third nucleon in the medium [67-69]. This three-nucleon force contains two parameters that are fixed by requiring that the BHF calculation reproduces the energy and saturation density of the symmetric nuclear matter. For the NY and YY interactions, we employed the NSC97e and NSC97a models [51,52]. The reason behind the choice of these models is that both models result in the best predictions for the hypernuclear observables among the potentials constructed by the Nijmegen group. Three-body forces involving hyperons, i.e., forces of the type NNY, NYY, and YYY, have been ignored in the calculation due to the large uncertainties still existing about these kind of forces.

To solve Equations (8)-(10), one starts from a reasonable guess for the single-particle potentials and obtain initial value of the G-matrix from Equation (8), which is solved in a partial wave basis including contributions up to a total angular momentum $J=4$. Then the new single-particle potential is computed from Equation (10) and passed as an input to Equations (8)-(9). These iterations continue until the desired level of convergence is reached. Once a self-consistent solution of Equations (8)-(10) is obtained, the baryon contribution to the total energy density can be calculated simply as

$$
\varepsilon_{B}=n_{B} \sum_{B_{i}} \sum_{\vec{k}_{B_{i}}} n\left(k_{B_{i}}\right)\left(m_{B_{i}}+\frac{k_{B_{i}}^{2}}{2 m_{B_{i}}}+\frac{1}{2} \operatorname{Re}\left[U_{B_{i}}\left(k_{B_{i}}\right)\right]\right),
$$

where $n_{B}$ is the baryon number density. Adding to $\varepsilon_{B}$ the contribution from the noninteracting leptons, $\varepsilon_{L}$, the composition and the EOS of neutron star matter can then be obtained from the requirement of equilibrium under weak interaction processes, $\mu_{i}=b_{i} \mu_{n}-q_{i} \mu_{e}$ ( $b_{i}$ and $q_{i}$ denoting the baryon number and electric charge of the species $i$, respectively), and electric charge neutrality, $\sum_{i} q_{i} n_{i}=0$. The chemical potentials of various species and the pressure are computed from the usual thermodynamic relations, $\mu_{i}=\partial \varepsilon / \partial n_{i}$ and $P=n_{B} \partial \varepsilon / \partial n_{B}-\varepsilon$, where $\varepsilon=\varepsilon_{B}+\varepsilon_{L}$ is the total energy density. The EOS (pressure versus total energy density) and the particle fractions $x_{i}=n_{i} / n_{B}$ of the different species are shown respectively in panels (a) and (b) of Figure 1. Vertical dashed lines in Figure $1 \mathrm{~b}$ show the threshold densities for appearance of muons $\left(0.132 \mathrm{fm}^{-3}\right), \Sigma^{-}$-hyperons $\left(0.248 \mathrm{fm}^{-3}\right.$ 
for NSC97e and $0.278 \mathrm{fm}^{-3}$ for NSC97a), and $\Lambda$-hyperons $\left(0.584 \mathrm{fm}^{-3}\right.$ for NSC97e and $0.642 \mathrm{fm}^{-3}$ for NSC97a).
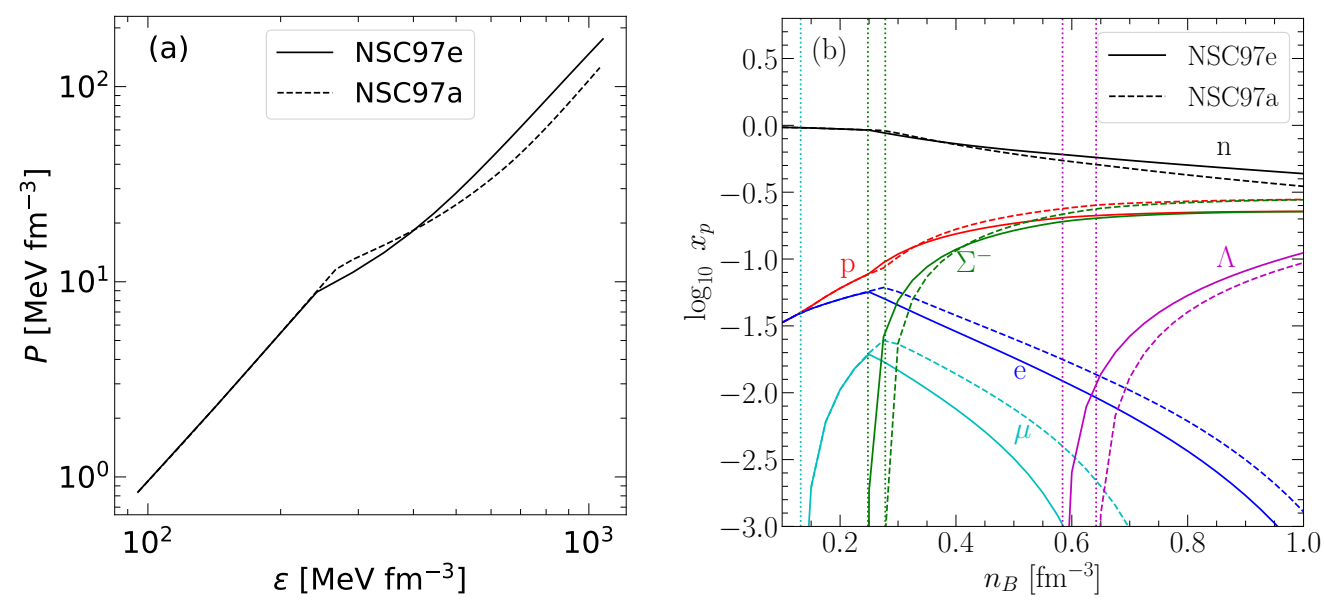

Figure 1. Properties of $\beta$-stable hypernuclear matter. Panel (a): Pressure as function of a total energy density for the NSC97e (solid lines) and NSC97a (dashed lines) models. Panel (b): Particle fractions as a function of total baryon number density for the NSC97e (solid lines) and NSC97a (dashed lines) models. Vertical dashed lines indicate $\mu, \Sigma^{-}$, and $\Lambda$ thresholds.

The G-matrices needed in the calculation of the transport-cross sections are taken on-shell and on the corresponding Fermi surfaces of the involved baryons, i.e., the starting energy is taken as $\omega=E_{B_{i}}\left(k_{F_{B_{i}}}\right)+E_{B_{j}}\left(k_{F_{B_{j}}}\right)$. The effective masses of the different baryons, required in the calculation of the transport coefficients, are calculated from their corresponding single-particle energies as

$$
\frac{m_{B_{i}}^{*}\left(k_{B_{i}}\right)}{m_{B_{i}}}=\frac{k_{B_{i}}}{m_{B_{i}}}\left(\frac{d E_{B_{i}}\left(k_{B_{i}}\right)}{d k_{B_{i}}}\right)^{-1} .
$$

Figure 2 shows the effective masses of the different baryons evaluated at each density at their corresponding Fermi momenta. The calculation of the effective masses via Equation (12) requires numerical differentiation of the single-particle potentials resulting from the solution of the BBG equations, which is a delicate numerical task. The results frequently contain considerable numerical noise. It is customary to approximate the results with some smooth function of the baryon density [70]. Here we employ this approach and fit $m_{c}^{*}\left(n_{B}\right)$ functions with fourth-order polynomials. The fit results are given in Appendix A.

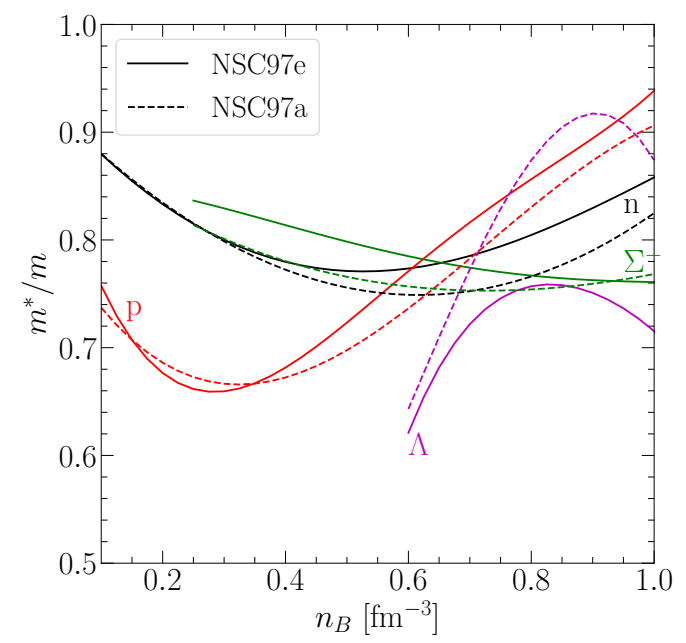

Figure 2. Effective baryon masses on the Fermi surface as a function of the total baryon number density for the NSC97e (solid lines) and NSC97a (dashed lines) models. 


\section{Results and Discussion}

\subsection{Transport Matrices and Mean Free Paths}

We have found that the results for the NSC97e and NSC97a models are qualitatively similar. Therefore for brevity we mainly focus on the NSC97e model until indicated otherwise.

The transport matrix $\Lambda_{c i}$ in Equation (4) contains a diagonal part [Equation (5)] and non-diagonal elements [Equation (6)]. If the non-diagonal elements can be neglected (as it is frequently the case, see below), the equations for the different species decouple and the effective mean free paths are given by a simple formula $\lambda_{c}=\Lambda_{c c}^{-1}$. The diagonal elements of the transport matrix thus represent useful approximations for the inverse mean free paths. In Figure 3 we show the diagonal components of the transport matrix for the baryon sector for thermal conductivity [panel (a)] and shear viscosity cases [panel (b)]. In this figure only the strong interaction channels are included and temperature-independent combinations $\Lambda_{c c} T_{8}^{-2}$ are shown, with $T_{8}=T /\left(10^{8} \mathrm{~K}\right)$. The behavior of $\Lambda_{c c}^{k}$ and $\Lambda_{c c}^{\eta}$ is qualitatively similar. The lowest values of $\Lambda_{c c}$ are obtained for neutrons. Thus, the neutrons are expected to have the largest mean free paths and dominate the baryon transport. The protons and $\Sigma^{-}$hyperons scatter one-two orders of magnitude more effectively, and the values of $\Lambda_{c c}$ for the $\Lambda$ hyperon lie in between. Notice almost identical results for protons and $\Sigma^{-}$hyperons in Figure 3 at $n_{B} \gtrsim 0.5 \mathrm{fm}^{-3}$.
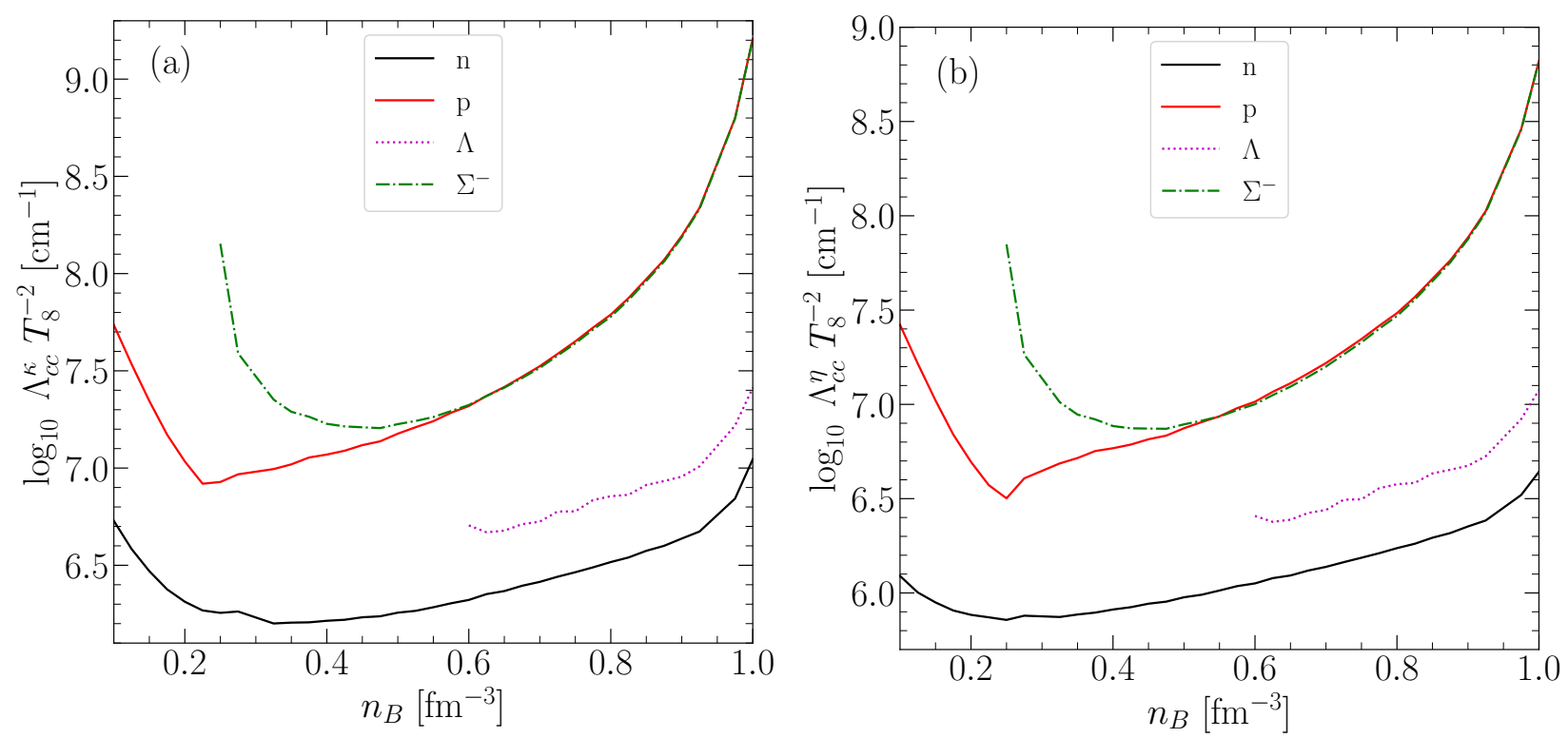

Figure 3. Diagonal elements of the transport matrix for baryons as a function of the total baryon number density. Different line types correspond to different particles, as indicated in the legend. Only the strong interaction is taken into account in this plot and temperature-independent $\left(\Lambda_{c c} T_{8}^{-2}\right)$ combinations are shown. Results are shown for the NSC97e model, respectively, for the thermal conductivity (panel (a)) and the shear viscosity cases (panel (b)).

These results can be understood by analyzing the partial contributions from the scattering on different species in Equation (5). In the matrix-shaped Figures 4 and 5 with the solid lines we plot the relative contributions to $\Lambda_{c c}$ for thermal conductivity and shear viscosity cases, respectively. The rows in these figures are numerated with index $c$ and columns with index $i$ of Equation (5). Namely, we show in each panel with solid lines the ratio $\widetilde{Y}_{c i}=\left(n_{i} \sigma_{c i}+\delta_{c i} n_{c} \sigma_{c c}^{\prime}\right) \Lambda_{c c}^{-1}$ (notice that the collisions of the like species contain additional $n_{c} \sigma_{c c}^{\prime}$ contribution). It is clear that soon after the $\Sigma^{-}$appearance, their mutual collisions with protons become completely dominant scattering mechanisms of these species. Taking into account that at $n_{B} \gtrsim 0.5 \mathrm{fm}^{-3}$ the proton and $\Sigma^{-}$number fractions are similar due to the requirement of charge neutrality in lack of charged leptons, see Figure $1 \mathrm{~b}$, the coincidence of their transport matrix elements in Figure 3 is natural. Because of the 
large values of the transport matrix elements, the proton and $\Sigma^{-}$mean free paths, $\lambda_{p}$ and $\lambda_{\Sigma^{-}}$, are small and, therefore, these two baryons are not expected to influence the transport equations for other species. Having this in mind and taking into account the relatively small fraction of these particles (in comparison to neutrons) in $\beta$-stable matter, one expects that they do not give sizable contribution to overall transport coefficients.
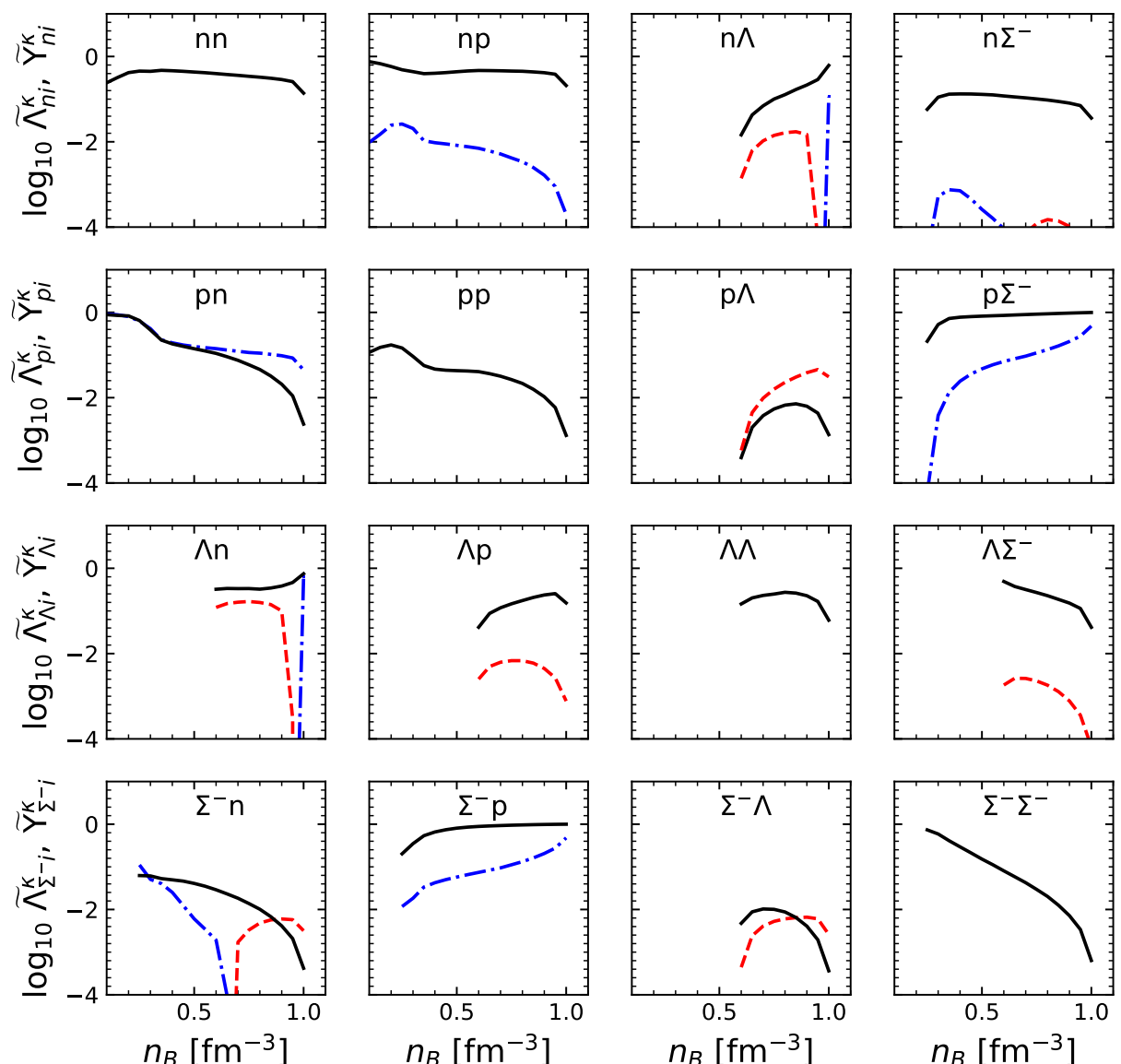

Figure 4. Normalized elements of the transport matrix for baryons for the thermal conductivity case and the NSC97e model. Solid lines in each row $\left(c=n, p, \Lambda, \Sigma^{-}\right)$show relative contributions $\widetilde{Y}_{c i}^{\kappa}$ to diagonal transport matrix elements from collisions with different baryon species. Other lines show the non-diagonal relative matrix elements $\widetilde{\Lambda}_{c i}^{\kappa}$, where red dashed lines correspond to positive values and the blue dash-dotted ones to negative values, respectively. Only strong forces are included, see text for details.

To analyze the mutual impact of the mean free paths of different species, it is instructive to introduce the renormalized mean free paths defined as $\widetilde{\lambda}_{c}=\lambda_{c} \Lambda_{c c}$. Then the system (4) takes the form

$$
1=\sum_{i} \frac{\Lambda_{c i}}{\Lambda_{i i}} \widetilde{\lambda}_{i} \equiv \sum_{i} \widetilde{\Lambda}_{c i} \widetilde{\lambda}_{i}
$$

where the renormalized transport matrix elements $\widetilde{\Lambda}_{c i}=\Lambda_{c i} / \Lambda_{i i}$ are introduced.

With red dashed (for positive values) and blue dash-dotted (for negative values) lines in Figures 4 and 5 we show logarithms of the non-diagonal relative matrix elements $\log _{10}\left|\widetilde{\Lambda}_{c i}\right|, c \neq i$. In the majority of cases, $\left|\widetilde{\Lambda}_{c i}\right| \ll 1$, which means that the influence of $\lambda_{i}$ on the equations for the $c$ species in that cases is negligible. Please note that irrespectively of the values of $\left|\widetilde{\Lambda}_{c i}\right|$ where one of the indices refers to protons or $\Sigma^{-}$hyperons, the corresponding terms can be safely neglected since $\lambda_{p}$ and $\lambda_{\Sigma^{-}}$are small as discussed above. This means that protons and $\Sigma^{-}$hyperons can be treated as passive scatterers. The remaining $\widetilde{\Lambda}_{c i}$ matrix elements correspond to the $n \Lambda$ subsystem. One observes that $\widetilde{\Lambda}_{\Lambda n}^{\eta}$ 
and $\widetilde{\Lambda}_{n \Lambda}^{\eta}$ and also, for the thermal conductivity case and at the largest densities, $\widetilde{\Lambda}_{\Lambda n}^{\kappa}$ and $\widetilde{\Lambda}_{n \Lambda}^{\kappa}$ are significant and may need to be taken into account.
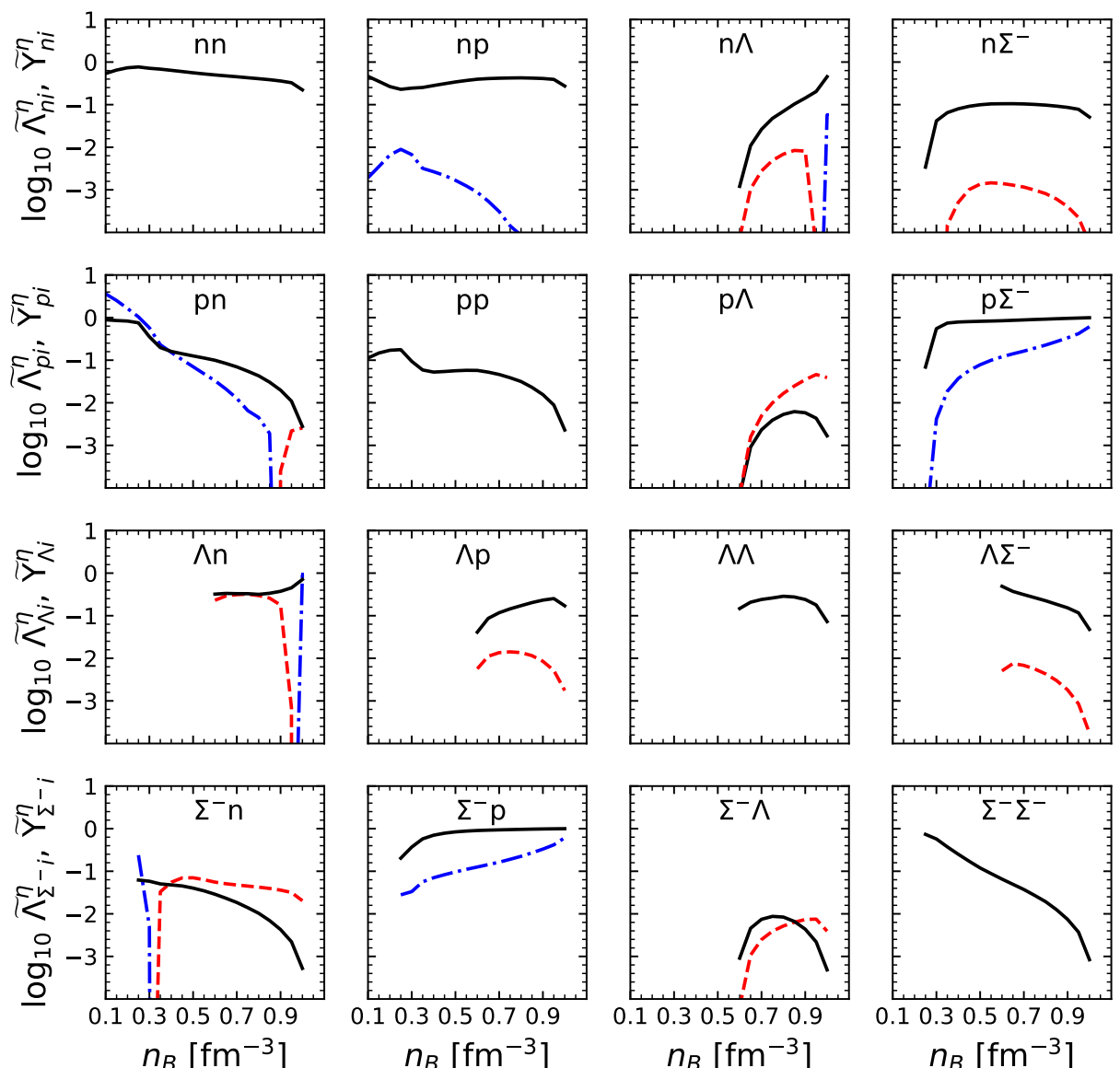

Figure 5. Normalized elements of the transport matrix for baryons for the shear viscosity case and NSC97e model. Solid lines in each row $\left(c=n, p, \Lambda, \Sigma^{-}\right)$show relative contributions $\widetilde{Y}_{c i}^{\eta}$ to diagonal transport matrix elements. Other lines show the non-diagonal relative matrix elements $\widetilde{\Lambda}_{c i}^{\eta}$, where red dashed lines correspond to positive values and the blue dash-dotted ones to negative values, respectively. Only strong forces are included, see text for details.

Up to now, we discussed the collisions in the baryon subsystem mediated by the strong interaction. The charged particles, $p \Sigma^{-} e \mu$ in our case, also participate in the electromagnetic interactions ${ }^{1}$. We neglect the interference between the electromagnetic and strong amplitudes and consider these interaction channels separately. The important difference between the mean free paths governed by electromagnetic and strong interactions in NS cores is in the non-Fermi-liquid temperature dependence of the former. This stems from the dynamical character of the plasma screening in the dominant transverse channel of the electromagnetic interaction [55,56]. As already stated, the matrix elements of the 'electromagnetic' transport matrix do not follow the same $\propto T^{2}$ scaling as the matrix elements for the strong interaction. For shear viscosity the contribution of the electromagnetic interactions of baryons is several orders of magnitude smaller than the contribution of strong interactions and, therefore, it can be always neglected, see e.g., Ref. [47]. For the thermal conductivity case at low temperatures, the diagonal elements of the transport matrix of charged particles are found to be given by a simple universal expression

$$
\Lambda_{c c}^{\kappa, e m}=\frac{6 \zeta(3)}{\pi^{2}} \alpha_{f} T=2.45 \times 10^{6} T_{8} \mathrm{~cm}^{-1},
$$


irrespectively of the content of the charged particles in the matter. For protons and $\Sigma^{-}$ hyperons at sufficiently low temperatures (e.g., $T \lesssim 10^{7} \mathrm{~K}$ ) it can become comparable with $\Lambda_{c c}^{\kappa}$ resulting from strong interactions. This makes the mean free paths of $p$ and $\Sigma^{-}$ even smaller so that they can be obtained by treating the baryon subsystem separately. Furthermore, non-diagonal matrix elements of $\Lambda_{c i}^{e m}$ are small both for viscosity and thermal conductivity [47]. As a consequence, lepton and baryon subsystems can indeed be treated separately. Moreover, in both subsystems $p$ and $\Sigma^{-}$act as passive scatterers. The influence of their non-equilibrium distributions on transport properties of other constituents is negligible. The combined contribution of $p$ and $\Sigma^{-}$to the total values of transport coefficients is found to be less than $10 \%$ for thermal conductivity and less then $5 \%$ for shear viscosity. In what follows, the transport coefficients of leptons are calculated according to Refs. [71,72].

\subsection{Corrections to the Variational Solution}

The variational solution gives a first approximation to the transport coefficients. In a multicomponent Fermi-liquid it is possible to obtain the exact solutions of the corresponding system of transport equations [44,45]. However, because of the non-Fermi-liquid behavior of the matrix elements of the electromagnetic interaction, obtaining the exact solution for the full $n p \Sigma^{-} \Lambda e \mu$ mixture is complicated and standard approaches $[44,45]$ do not work. Nevertheless, as discussed above, it is sufficient to consider the $n \Lambda$ and $e \mu$ subsystems separately, with protons and $\Sigma^{-}$particles acting as the passive scatterers. The corrections to the variational solution in the electromagnetic sector were considered in Refs. [71,72] and were found to be less than $5-10 \%$.

The corrections to the variational solution in the $n \Lambda$ subsystem can be calculated using the method described in Ref. [45], or by the numerical solution of the system of integral equations [53]. It is customary to express transport coefficients resulting from the exact solution, via the correction coefficients

$$
\kappa_{\mathrm{ex}}=C_{\mathrm{ex}}^{\kappa} \kappa_{\mathrm{var}}, \quad \eta_{\mathrm{ex}}=C_{\mathrm{ex}}^{\eta} \eta_{\mathrm{var}} .
$$

In Figure 6 we show the correction factors $C_{\mathrm{ex}}$ for the thermal conductivity (solid line) and shear viscosity (dashed line) for the $n \Lambda$ subsystem, where protons and $\Lambda$-hyperons are treated as passive scatterers. The results are similar to the nuclear matter $[46,47,53]$. For the shear viscosity, the correction coefficient does not exceed $5 \%$; this correction is truly minor having in mind other systematic uncertainties and it can be neglected. The correction coefficient for the thermal conductivity case is about $20 \%$ for the $n \Lambda$ and can be included in calculations.

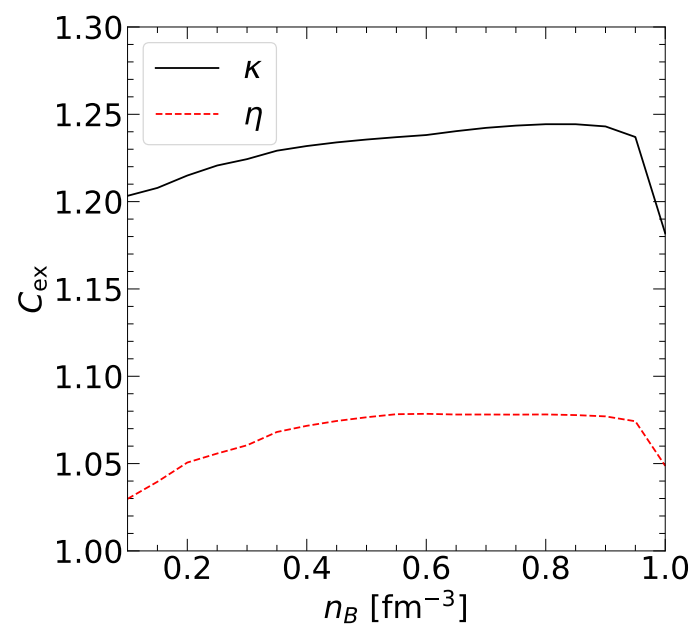

Figure 6. Corrections to the variational solution for the $n \Lambda$ subsystem (obtained with the NSC97e model). Solid and dashed lines show correction coefficients for the thermal conductivity and shear viscosity cases, respectively. 
We do not discuss the corrections to the simplest approximation for the momentum transfer rates $J_{c i}$. These quantities are required as an input to the studies of the magnetic field evolution in NS cores. In this case the exact solution of the transport equations in the presence of the magnetic field is required. Such solutions have never been constructed for multicomponent Fermi-systems to the best of our knowledge and require a separate study. Since similar transport cross-sections (with the same leading-order dependencies) enter expressions for the shear viscosity and the momentum transfer rates, one can expect that the corrections resulting from better approximations to the transport equations will not be large.

\subsection{Transport Coefficients}

Figures 7 and 8 show, respectively, the partial contributions of the matter constituents to the thermal conductivity and shear viscosity coefficients. These contributions are obtained via the simplest variational solution. The results are shown for the temperatureindependent (in a normal Fermi-liquid) combinations $\kappa T_{8}$ and $\eta T_{8}^{2}$. Due to the non-Fermi liquid behavior of the lepton transport coefficients, we show results for three temperature values, $T=10^{7}, 10^{8}$, and $10^{9} \mathrm{~K}$. Thick solid lines in Figures 7 and 8 show total thermal conductivity and shear viscosity coefficients, respectively, including the corrections described in Section 4.2.
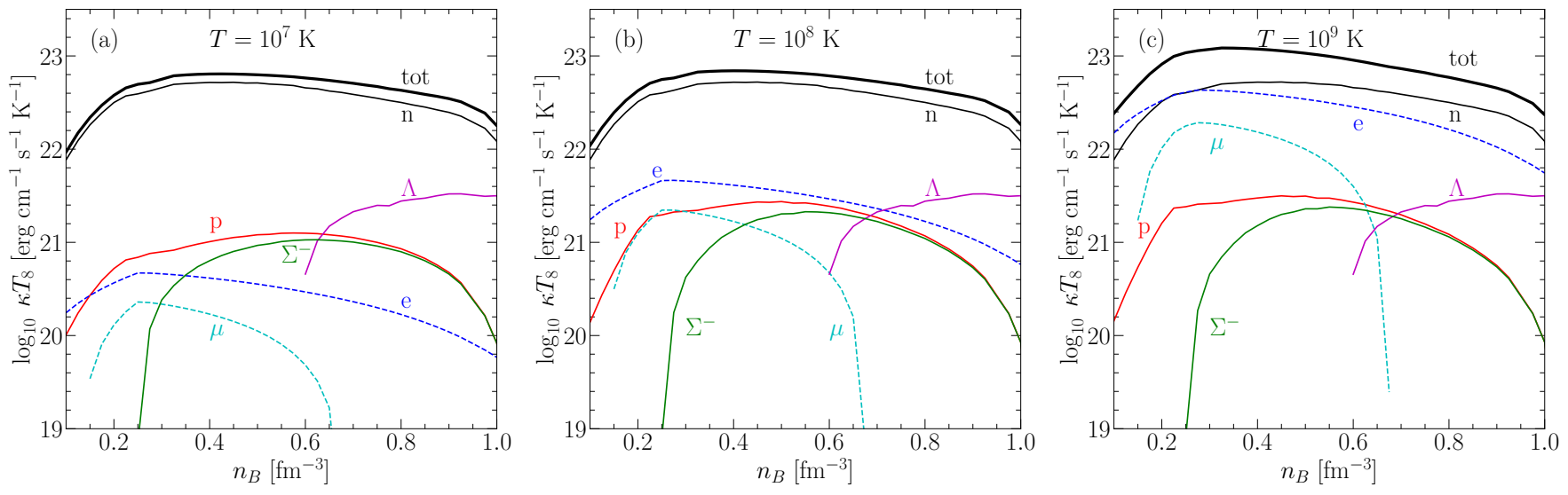

Figure 7. Partial contributions to the thermal conductivity coefficient (variational solution) for the $\beta$-stable matter in NS cores. Results for the NSC97e model are shown. Particle species labels are shown near the corresponding curves. The total thermal conductivity including corrections to the variational solution is shown with the thick solid line. Results for three values of the temperature are shown: $T=10^{7} \mathrm{~K}(\mathbf{a}), T=10^{8} \mathrm{~K}(\mathbf{b})$ and $T=10^{9} \mathrm{~K}(\mathbf{c})$.
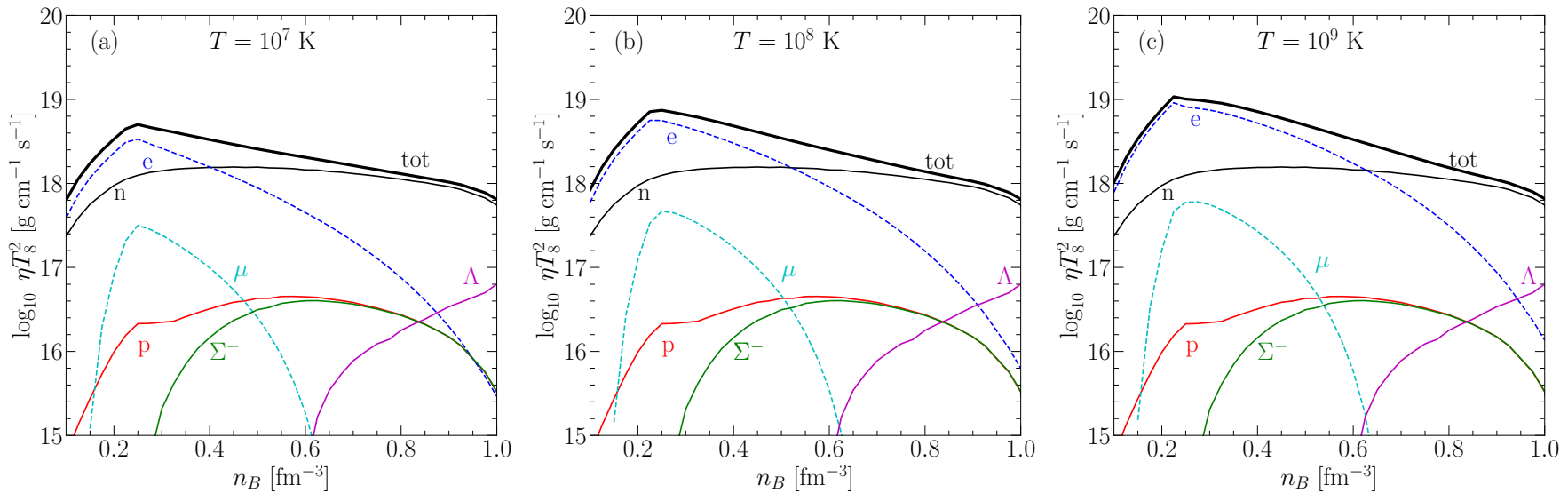

Figure 8. Partial contributions to the shear viscosity coefficient (variational solution) for the beta-stable mater in NS cores. Results for the NSC97e model are shown. Particle species labels are shown near the corresponding curves. The total shear viscosity including corrections to the variational solution is shown with the thick solid line. Results for three values of the temperature are shown: $T=10^{7} \mathrm{~K}(\mathbf{a}), T=10^{8} \mathrm{~K}(\mathbf{b})$ and $T=10^{9} \mathrm{~K}(\mathbf{c})$. 
Among the baryon sector, neutrons always dominate the transport, such as in the case of nuclear matter $[46,47]$. In the case of the thermal transport, neutrons dominate also the total value of the thermal conductivity except for $T=10^{9} \mathrm{~K}$ where the lepton contribution becomes comparable to the nucleon one, see Figure 7c. This behavior is similar to that found in the nucleonic NS cores [46,47].

A different situation is observed in the case of the shear viscosity, see Figure 8 . Here the leptonic contribution is dominant at lower densities (the upper boundary of this region depends on temperature), while neutrons give the main contribution at higher densities due to decrease in the lepton number fractions induced by the onset of the $\Sigma^{-}$hyperon. This is in contrast with the results of the nucleonic matter where the lepton contribution was found to be always dominant in the non-superfluid matter [46,47]. The total baryon contribution to $\eta$ is similar for both nucleon and hyperon NS core compositions. However, as a result of the suppression of lepton contribution, the total shear viscosity of the hypernuclear matter can be several orders of magnitude smaller than those in the nuclear matter.

Let us investigate the model dependence of the obtained results. Comparing the results for the NSC9e and NSC97a potentials we find that although the partial transport coefficients exhibit some variations, the total values dominated by neutrons and leptons do not change much. This is illustrated in Figure 9, where we show thermal conductivity (panel (a)) and shear viscosity (panel (b)) for the NSC97e (solid lines) and NSC97a (dashed lines) for three values of temperature as indicated in the plot. Notice that here the absolute values of $\kappa$ and $\eta$ (without temperature rescaling) are plotted for convenience. The difference between the two models is not large. The reason is that the NSC97e and NSC97a models for the NY and YY interactions are quite similar and differ only on the values of a few meson-baryon coupling constants, cut-off values of the vertex form factors, and some other parameters. Both describe, as mentioned before, with the same accuracy the existing hypernuclear data. In some sense this resembles the results for the nuclear matter of Ref. [47], where it was found that the main source for difference between the various models originates from the model for the three-body interactions which we do not include here for hyperons and do not alter for nucleons. Neutrons, as the most significant particles in terms of transport properties, are not affected by the change of model. The situation is different for other particles, see below.
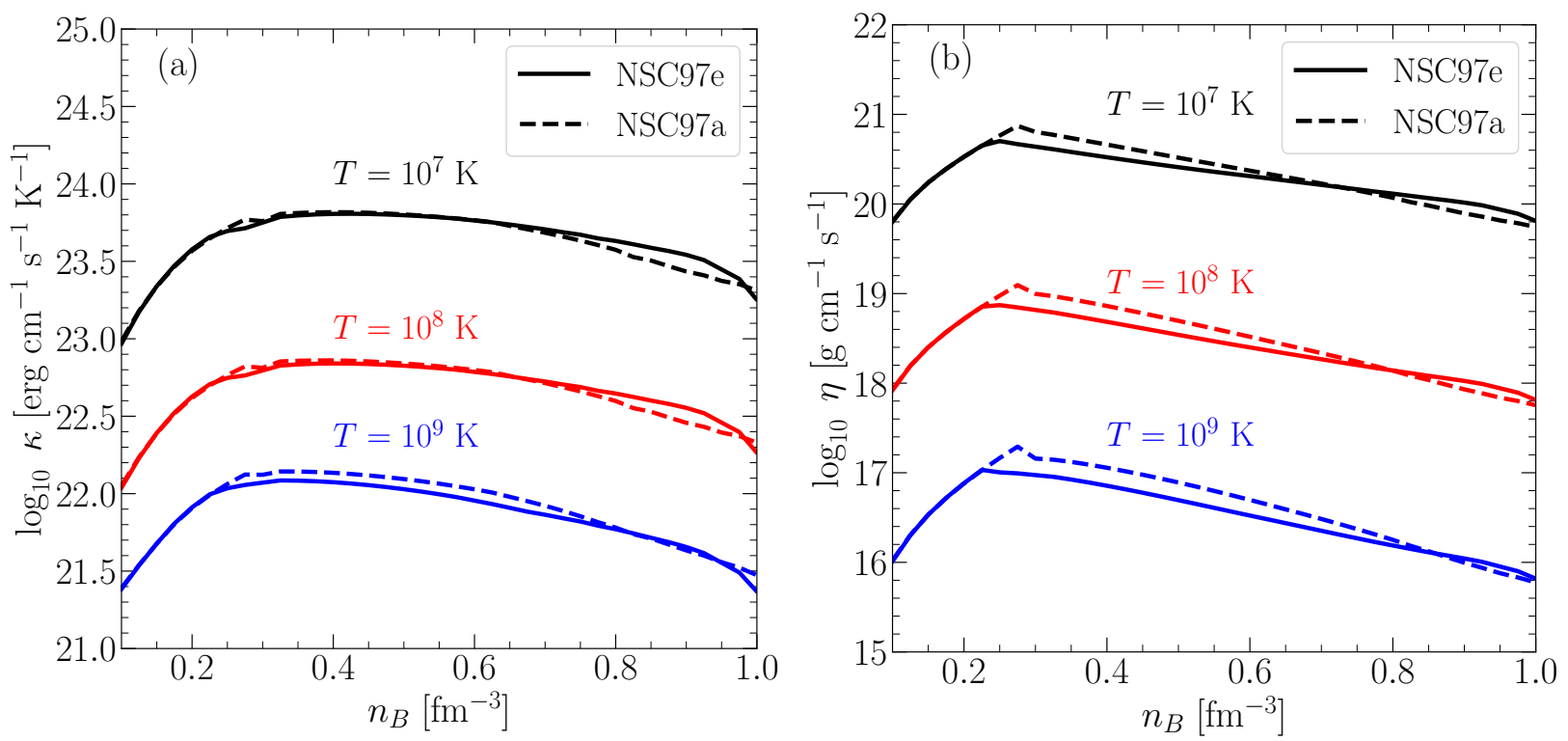

Figure 9. Total thermal conductivity (a) and shear viscosity (b) in hypernuclear NS cores for the NSC97e (solid lines) and NSC97 (dashed lines) models. Here we do not perform temperature rescaling of the transport coefficients. The results are shown for three values of temperature, $T=10^{7}, 10^{8}$, and $10^{9} \mathrm{~K}$ as indicated in the plot. 
Finally, in Figure 10 we show the momentum transfer rates in the baryon collisions. Panel (a) corresponds to the NSC97e model and panel (b) to the NSC97a model. Here, we plot the temperature-independent combination $J_{c i} T_{8}^{-2}$. The contribution of the electromagnetic interactions to $J_{c i}$ is negligible like in the case of the shear viscosity above. The momentum transfer rates mediated by the electromagnetic interactions can be calculated following the expressions in Refs. [42,73]. They are several orders of magnitude smaller than $J_{c i}$ mediated by the strong interaction, and we do not show them here. The momentum transfer rate between the nucleons, $J_{n p}$, is in an order-of-magnitude agreement with the results of the pure nucleonic matter [47]. A large value of $J_{p \Sigma^{-}}$draws attention in Figure 10. This is due to the particularly strong interaction in this channel predicted by the NSC97 models, see Refs. [51,52]. Notice that for this transport coefficient the difference between the NSC97e and NSC97a model becomes apparent.
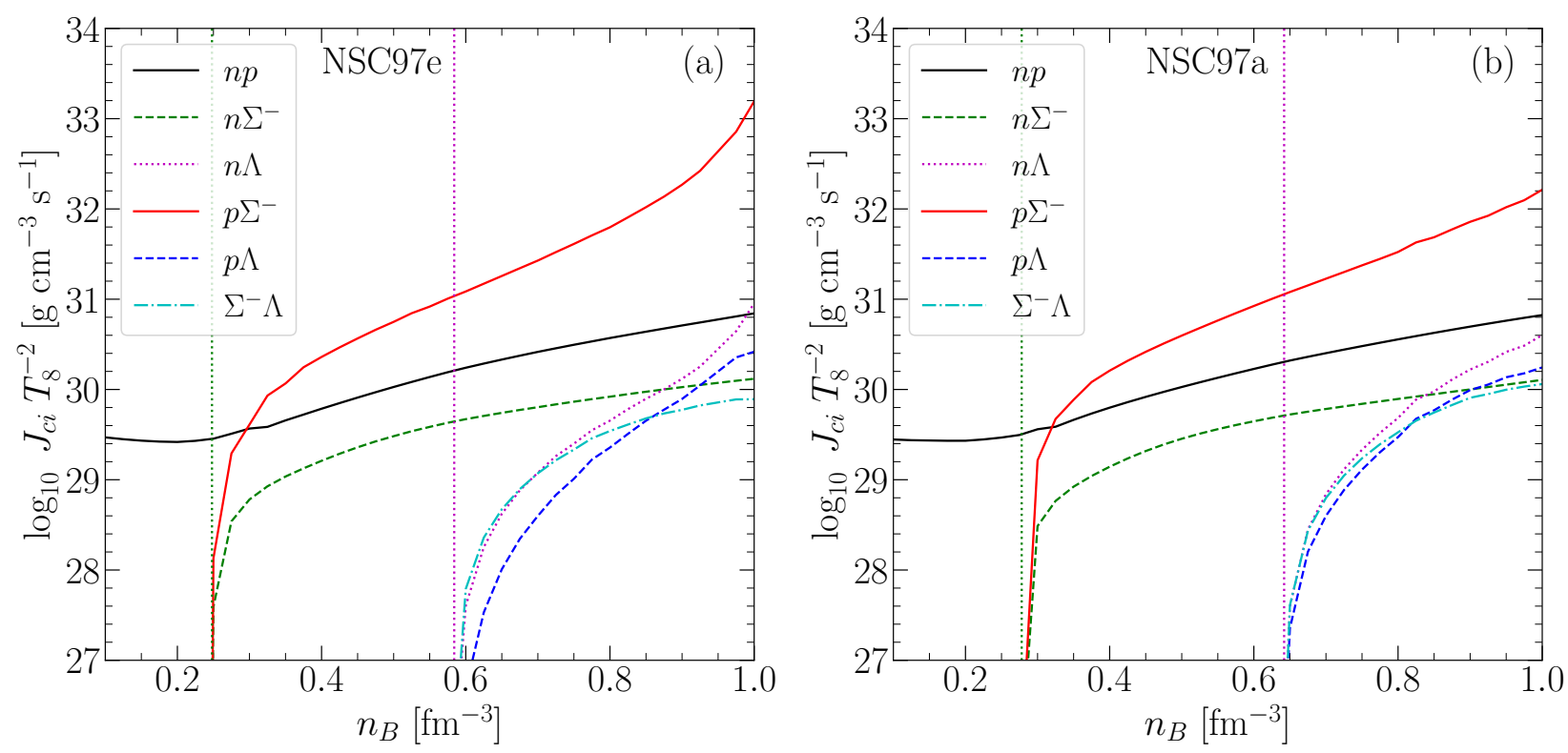

Figure 10. Momentum transfer rates $J_{c i}$ mediated by the strong interactions for the NSC97e (panel a) and NSC97a (panel b) models. The temperature-independent combination $J_{c i} T_{8}^{-2}$ is shown. Different lines correspond to different baryon pairs as shown in the legend. Vertical dashed lines indicate $\Sigma^{-}$and $\Lambda$ thresholds.

To facilitate the application of our study, we give the numerical results for the total thermal conductivity and shear viscosity coefficients, as well as the momentum transfer rates in Tables A3 and A4 in Appendix B.

\section{Conclusions}

In this work, we calculated the transport coefficients of the hypernuclear matter in the NS cores. In particular, we calculated the thermal conductivity, the shear viscosity, and the momentum transfer rates for $\mathrm{np} \Sigma^{-} \Lambda e \mu$ matter in $\beta$-equilibrium for baryon number densities between 0.1 and $1 \mathrm{fm}^{-3}$. The hypernuclear matter EOS was described within the non-relativistic BHF approach using realistic NN, NY, and YY interactions. Our results have shown that the proton, $\Sigma^{-}$, and $\Lambda$ do not give sizable contributions to the overall transport coefficients, being the total baryon contribution dominated by the neutron one as in the case of neutron star cores with only nucleons. However, these species are important in mediating the neutron mean free path. In particular, we found that neutrons dominate also the total thermal conductivity over the whole range of densities explored and that, due to the onset of $\Sigma^{-}$which leads to the deleptonization of the neutron star core, they dominate also the shear viscosity on the high density region, in contrast with the pure nucleonic case where the lepton contribution is always the dominant one. 
Our paper makes only a first step in the studies of the transport properties of hypernuclear NS cores with realistic baryon interactions. The present study can be improved and extended in various aspects.

In particular, we employed particular potentials both for nucleon and strange sectors and a specific many-body theory. Based on the experience for the nuclear sector [47], we expect that the variations of baryon interaction can lead to a substantial modifications of the values of the transport coefficients at large (e.g., $\gtrsim 2 n_{0}$ ) baryon densities, especially when the three-body interactions are altered. Nevertheless, the qualitative conclusions presented above should be stable over the choice of a particular strong interaction model, which is seen comparing NSC97e and NSC97a results.

Next, the neutron star cores can contain strong magnetic fields. In this case the transport becomes anisotropic due to the Larmor rotation of the charged particles. The inclusion of these effects in not too strong magnetic fields, when the transport cross-sections are not modified, is relatively straightforward. For instance, the momentum transfer rates as calculated here constitute the microphysical input for the magnetic field evolution studies, see, e.g., Ref. [41,42]. Large fields, e.g., $\gtrsim 10^{14} \mathrm{G}$, may affect the polarization tensor of the plasma making the scattering of the charged particles due to electromagnetic interactions anisotropic. This case is more complicated and requires a separate study. In even stronger fields, e.g., $\gtrsim 10^{18} \mathrm{G}$, the particle motion becomes strongly quantized and the description of the transport based on the semiclassical distribution functions becomes invalid; however the presence of such ultra-high magnetic fields can hardly be considered to be realistic.

Finally, due to the presence of the attractive character of some partial wave channels of the baryon interaction, the baryon can be subject to pairing instabilities, see, e.g., Ref. [74], for a review. Naturally, the strength and the character of the particular partial wave interaction is density-dependent. It is expected that the protons in NS cores pair in the ${ }^{1} \mathrm{~S}_{0}$ channel, while the neutrons should pair in the ${ }^{3} \mathrm{P}_{2}-{ }^{3} \mathrm{~F}_{2}$ channel. Hyperons may also form superfluids if their interactions are attractive enough. However, a quantitative estimation of the hyperon pairing, contrary to the nucleonic one, has not received so much attention and just a few calculations exist in the literature [75-81]. In the presence of the paired species, the dynamical processes in NSs are described by a complicated multifluid hydrodynamics of the superfluid/suprerconducting mixtures e.g., [82], and references therein. Baryon pairing affects the transport coefficients of the NS core matter in several ways (see, e.g., [39], for review). We briefly outline the main effects expected in the presence of pairing. In this case the transport of the normal component of matter is governed by the Bogoliubov quasiparticles, which spectra have a gap at the Fermi surface. This results in exponential suppression of the scattering rates of quasiparticles at low temperatures. In NS core context, this effect was considered in Ref. [83] for neutron thermal conductivity and in Refs. [71,72] for lepton transport coefficients in the presence of the proton pairing. Moreover, the collision integral for the Bogouluibov quasiparticles modifies and should include processes with non-conserved number of quasiparticles, e.g., [84]. In addition, if charged species are paired, the electromagnetic polarization of this superconducting matter is modified which affects the scattering rate of all charged quasiparticles (even non-superconducting ones, such as leptons) [39,47]. At low temperatures, the gapless Goldstone modes, e.g., superfluid phonons, can contribute to the transport properties of matter, see, e.g., [85], for a review.

Even more complicated is the case of the magnetized superfluid/superconducting matter, where-if the type II superconductivity is realized-the topological structures called Abrikosov vortices appear. The study of all these effects is much desirable but is beyond the scope of the present paper.

Author Contributions: Both authors contributed equally to this work. All authors have read and agreed to the published version of the manuscript. 
Funding: A part of this work was done during the PHAROS COST STSM \#CA16214-44662. The work was partially supported by the Foundation for the Advancement of Theoretical Physics and Mathematics “BASIS”, grant 17-13-205-1.

Data Availability Statement: The data presented in this study are available on request from the corresponding author

Acknowledgments: We thank the anonymous referees for suggestions which helped us to improve the paper. P.S. Thanks the INFN Sez. di Catania for hospitality.

Conflicts of Interest: The authors declare no conflict of interest.

\section{Abbreviations}

The following abbreviations are used in this manuscript:

NS Neutron star

EOS Equation of state

BHF Brueckner-Hartree-Fock

Av18 Argonne v18

UIX Urbana IX

NSC97 Nijmegen Soft-Core 97

\section{Appendix A} sion

We fit the dependence of effective masses on baryon density via the following expres-

$$
\frac{m_{c}^{*}}{m_{c}}=\sum_{k=0}^{4} a_{c k}\left(\frac{n_{B}}{1 \mathrm{fm}^{-3}}\right)^{k}
$$

for $c=n, p, \Sigma^{-}$, and $\Lambda$. The fit coefficients $a_{c k}$ are given in Table A1 for NSC97e model and in Table A2 for NSC97a model. Notice that in the latter case we used third-order polynomials.

Table A1. Coefficients of the polynomial fit (A1) for NSC97e model. Last column gives the density range where the expression (A1) is valid.

\begin{tabular}{lrrrrrl}
\hline Species & $\boldsymbol{a}_{\mathbf{0}}$ & $\boldsymbol{a}_{\mathbf{1}}$ & $\boldsymbol{a}_{\mathbf{2}}$ & $\boldsymbol{a}_{\mathbf{3}}$ & $\boldsymbol{a}_{\mathbf{4}}$ & $\boldsymbol{n}_{\boldsymbol{B}}$ Range \\
\hline$n$ & 0.940 & -0.668 & 0.678 & 0.029 & -0.121 & $0.10-1 \mathrm{fm}^{-3}$ \\
$p$ & 0.933 & -2.343 & 6.504 & -6.472 & 2.316 & $0.10-1 \mathrm{fm}^{-3}$ \\
$\Sigma^{-}$ & 0.853 & 0.0418 & -0.590 & 0.695 & -0.239 & $0.25-1 \mathrm{fm}^{-3}$ \\
$\Lambda$ & -4.256 & 19.554 & -28.78 & 19.27 & -5.077 & $0.60-1 \mathrm{fm}^{-3}$ \\
\hline
\end{tabular}

Table A2. Coefficients of the polynomial fit (A1) for NSC97a model. Last column gives the density range where the expression (A1) is valid. Notice that the $a_{4}$ coefficient is absent.

\begin{tabular}{lrrrrl}
\hline Species & $a_{\mathbf{0}}$ & $\boldsymbol{a}_{\mathbf{1}}$ & $\boldsymbol{a}_{\mathbf{2}}$ & $\boldsymbol{a}_{\mathbf{3}}$ & $\boldsymbol{n}_{\boldsymbol{B}}$ Range \\
\hline$n$ & 0.934 & -0.591 & 0.453 & 0.029 & $0.10-1 \mathrm{fm}^{-3}$ \\
$p$ & 0.826 & -1.092 & 2.171 & -1.000 & $0.10-1 \mathrm{fm}^{-3}$ \\
$\Sigma^{-}$ & 0.905 & -0.459 & 0.401 & -0.078 & $0.30-1 \mathrm{fm}^{-3}$ \\
$\Lambda$ & 0.892 & -3.916 & 8.742 & -4.844 & $0.65-1 \mathrm{fm}^{-3}$ \\
\hline
\end{tabular}

\section{Appendix B}

Tables A3 and A4 contain the numerical values of the transport coefficients for the hypernuclear NS core composition discussed in the main text for NSC97e and NSC97a models, respectively. We show $\kappa T_{8}$ (columns 2-4) and $\eta T_{8}^{2}$ (columns 5-7) for three values of the temperature. Total (lepton plus baryon) values of the transport coefficients are given. In the baryon subsystem the corrections for the variational solution for $n$ and $\Lambda$ are 
included. We also tabulate the momentum transfer rates $J_{c i} T_{8}^{-2}$ in collisions of six possible distinguishable baryon pairs (columns 7-13).

Table A3. Transport coefficients of the hypernuclear matter as a function of baryon density for the NSC97e model. Columns 2-4: total thermal conductivity for three values of temperature. Columns 5-7: total shear viscosity for three values of temperature. For $\eta$ and $\kappa$ exact solutions of transport equations in the sense described in the text are shown. Columns 8-14: momentum transfer rates $J_{c i}$.

\begin{tabular}{|c|c|c|c|c|c|c|c|c|c|c|c|c|}
\hline \multirow{3}{*}{$\begin{array}{c}n_{B} \\
\mathrm{fm}^{-3}\end{array}$} & \multirow{2}{*}{\multicolumn{3}{|c|}{ 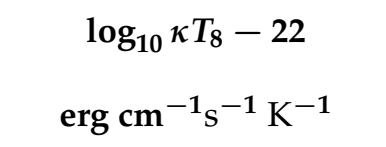 }} & \multirow{2}{*}{\multicolumn{3}{|c|}{$\begin{array}{c}\log _{10} \eta T_{8}^{2}-18 \\
\mathrm{~g} \mathrm{~cm}^{-1} \mathrm{~s}^{-1}\end{array}$}} & \multirow{2}{*}{\multicolumn{6}{|c|}{$\begin{array}{c}\log _{10} J_{c i} T_{8}^{-2}-29 \\
\mathrm{~g} \mathrm{~cm}^{-3} \mathrm{~s}^{-1}\end{array}$}} \\
\hline & & & & & & & & & & & & \\
\hline & $10^{7} \mathrm{~K}$ & $10^{8} \mathrm{~K}$ & $10^{9} \mathrm{~K}$ & $10^{7} \mathrm{~K}$ & $10^{8} \mathrm{~K}$ & $10^{9} \mathrm{~K}$ & $n p$ & $n \Sigma^{-}$ & $n \Lambda$ & $p \Sigma^{-}$ & $p \Lambda$ & $\Sigma^{-} \Lambda$ \\
\hline 0.100 & -0.032 & 0.038 & 0.384 & -0.201 & -0.078 & 0.012 & 0.469 & - & - & - & - & - \\
\hline 0.125 & 0.175 & 0.230 & 0.537 & 0.051 & 0.192 & 0.302 & 0.449 & - & - & - & - & - \\
\hline 0.150 & 0.340 & 0.392 & 0.678 & 0.24 & 0.401 & 0.532 & 0.433 & - & - & - & - & - \\
\hline 0.175 & 0.472 & 0.523 & 0.807 & 0.392 & 0.570 & 0.719 & 0.423 & - & - & - & - & - \\
\hline 0.200 & 0.578 & 0.627 & 0.912 & 0.528 & 0.719 & 0.883 & 0.418 & - & - & - & - & - \\
\hline 0.225 & 0.657 & 0.707 & 0.995 & 0.651 & 0.854 & 1.032 & 0.431 & - & - & - & - & - \\
\hline 0.250 & 0.696 & 0.748 & 1.035 & 0.702 & 0.871 & 1.004 & 0.455 & -1.407 & - & -0.869 & - & - \\
\hline 0.275 & 0.714 & 0.763 & 1.056 & 0.668 & 0.845 & 0.993 & 0.510 & -0.461 & - & 0.292 & - & - \\
\hline 0.300 & 0.750 & 0.795 & 1.071 & 0.639 & 0.817 & 0.974 & 0.567 & -0.219 & - & 0.611 & - & - \\
\hline 0.325 & 0.787 & 0.827 & 1.085 & 0.611 & 0.789 & 0.955 & 0.586 & -0.073 & - & 0.933 & - & - \\
\hline 0.350 & 0.796 & 0.834 & 1.084 & 0.580 & 0.755 & 0.924 & 0.657 & 0.036 & - & 1.067 & - & - \\
\hline 0.375 & 0.803 & 0.839 & 1.080 & 0.550 & 0.720 & 0.891 & 0.722 & 0.126 & - & 1.246 & - & - \\
\hline 0.400 & 0.806 & 0.841 & 1.074 & 0.521 & 0.684 & 0.855 & 0.787 & 0.210 & - & 1.360 & - & - \\
\hline 0.425 & 0.807 & 0.840 & 1.065 & 0.493 & 0.648 & 0.817 & 0.849 & 0.288 & - & 1.466 & - & - \\
\hline 0.450 & 0.806 & 0.837 & 1.055 & 0.465 & 0.612 & 0.777 & 0.910 & 0.359 & - & 1.565 & - & - \\
\hline 0.475 & 0.803 & 0.832 & 1.043 & 0.437 & 0.575 & 0.736 & 0.970 & 0.425 & - & 1.661 & - & - \\
\hline 0.500 & 0.798 & 0.826 & 1.029 & 0.410 & 0.539 & 0.694 & 1.028 & 0.483 & - & 1.749 & - & - \\
\hline 0.525 & 0.792 & 0.819 & 1.013 & 0.384 & 0.503 & 0.651 & 1.083 & 0.537 & - & 1.845 & - & - \\
\hline 0.550 & 0.785 & 0.810 & 0.996 & 0.359 & 0.468 & 0.608 & 1.138 & 0.586 & - & 1.918 & - & - \\
\hline 0.575 & 0.776 & 0.800 & 0.977 & 0.335 & 0.433 & 0.565 & 1.191 & 0.631 & - & 2.006 & - & - \\
\hline 0.600 & 0.764 & 0.786 & 0.955 & 0.311 & 0.399 & 0.522 & 1.241 & 0.671 & -1.398 & 2.086 & -2.312 & -1.210 \\
\hline 0.625 & 0.752 & 0.773 & 0.933 & 0.287 & 0.366 & 0.480 & 1.287 & 0.707 & -0.746 & 2.172 & -1.470 & -0.641 \\
\hline 0.650 & 0.738 & 0.757 & 0.908 & 0.262 & 0.333 & 0.437 & 1.331 & 0.741 & -0.378 & 2.257 & -0.992 & -0.328 \\
\hline 0.675 & 0.722 & 0.740 & 0.884 & 0.238 & 0.300 & 0.395 & 1.374 & 0.773 & -0.118 & 2.343 & -0.655 & -0.103 \\
\hline 0.700 & 0.707 & 0.724 & 0.864 & 0.213 & 0.267 & 0.352 & 1.417 & 0.804 & 0.078 & 2.428 & -0.400 & 0.071 \\
\hline 0.725 & 0.688 & 0.705 & 0.842 & 0.188 & 0.234 & 0.310 & 1.456 & 0.835 & 0.262 & 2.522 & -0.169 & 0.216 \\
\hline 0.750 & 0.672 & 0.688 & 0.820 & 0.164 & 0.203 & 0.269 & 1.495 & 0.865 & 0.400 & 2.612 & 0.010 & 0.334 \\
\hline 0.775 & 0.648 & 0.664 & 0.793 & 0.138 & 0.171 & 0.227 & 1.533 & 0.893 & 0.548 & 2.707 & 0.223 & 0.459 \\
\hline 0.800 & 0.632 & 0.647 & 0.770 & 0.115 & 0.141 & 0.188 & 1.570 & 0.920 & 0.655 & 2.797 & 0.357 & 0.540 \\
\hline 0.825 & 0.610 & 0.625 & 0.744 & 0.090 & 0.111 & 0.150 & 1.605 & 0.948 & 0.782 & 2.907 & 0.508 & 0.612 \\
\hline 0.850 & 0.588 & 0.601 & 0.715 & 0.065 & 0.082 & 0.113 & 1.640 & 0.975 & 0.897 & 3.019 & 0.654 & 0.680 \\
\hline 0.875 & 0.567 & 0.579 & 0.687 & 0.041 & 0.054 & 0.078 & 1.675 & 1.001 & 1.000 & 3.137 & 0.776 & 0.734 \\
\hline 0.900 & 0.542 & 0.554 & 0.656 & 0.015 & 0.025 & 0.043 & 1.709 & 1.026 & 1.118 & 3.269 & 0.895 & 0.774 \\
\hline 0.925 & 0.508 & 0.520 & 0.616 & -0.015 & -0.008 & 0.005 & 1.742 & 1.050 & 1.253 & 3.422 & 1.045 & 0.823 \\
\hline 0.950 & 0.448 & 0.459 & 0.554 & -0.062 & -0.057 & -0.047 & 1.776 & 1.073 & 1.449 & 3.639 & 1.200 & 0.857 \\
\hline 0.975 & 0.387 & 0.398 & 0.491 & -0.109 & -0.106 & -0.099 & 1.809 & 1.096 & 1.645 & 3.855 & 1.355 & 0.890 \\
\hline 1.000 & 0.251 & 0.264 & 0.368 & -0.190 & -0.187 & -0.183 & 1.843 & 1.118 & 1.954 & 4.192 & 1.418 & 0.892 \\
\hline
\end{tabular}


Table A4. Same as Table A3 but for the NSC97a model.

\begin{tabular}{|c|c|c|c|c|c|c|c|c|c|c|c|c|}
\hline \multirow{3}{*}{$\begin{array}{c}n_{B} \\
\mathrm{fm}^{-3}\end{array}$} & \multirow{2}{*}{\multicolumn{3}{|c|}{$\begin{array}{c}\log _{10} \kappa T_{8}-22 \\
\operatorname{erg} \mathrm{cm}^{-1} \mathrm{~S}^{-1} \mathrm{~K}^{-1}\end{array}$}} & \multirow{2}{*}{\multicolumn{3}{|c|}{$\begin{array}{c}\log _{10} \eta T_{8}^{2}-18 \\
\mathrm{~g} \mathrm{~cm}^{-1} \mathrm{~s}^{-1}\end{array}$}} & \multirow{2}{*}{\multicolumn{6}{|c|}{$\begin{array}{c}\log _{10} J_{c i} T_{8}^{-2}-29 \\
\mathrm{~g} \mathrm{~cm}^{-3} \mathrm{~s}^{-1}\end{array}$}} \\
\hline & & & & & & & & & & & & \\
\hline & $10^{7} \mathrm{~K}$ & $10^{8} \mathrm{~K}$ & $10^{9} \mathrm{~K}$ & $10^{7} \mathrm{~K}$ & $10^{8} \mathrm{~K}$ & $10^{9} \mathrm{~K}$ & $n p$ & $n \Sigma^{-}$ & $n \Lambda$ & $p \Sigma^{-}$ & $p \Lambda$ & $\Sigma^{-} \Lambda$ \\
\hline 0.100 & -0.015 & 0.052 & 0.391 & -0.193 & -0.07 & 0.022 & 0.446 & - & - & - & - & - \\
\hline 0.125 & 0.182 & 0.237 & 0.540 & 0.053 & 0.195 & 0.306 & 0.438 & - & - & - & - & - \\
\hline 0.150 & 0.338 & 0.39 & 0.677 & 0.239 & 0.401 & 0.532 & 0.435 & - & - & - & - & - \\
\hline 0.175 & 0.465 & 0.517 & 0.804 & 0.39 & 0.567 & 0.716 & 0.432 & - & - & - & - & - \\
\hline 0.200 & 0.568 & 0.619 & 0.907 & 0.525 & 0.716 & 0.88 & 0.432 & - & - & - & - & - \\
\hline 0.225 & 0.648 & 0.698 & 0.991 & 0.648 & 0.851 & 1.028 & 0.447 & - & - & - & - & - \\
\hline 0.250 & 0.715 & 0.766 & 1.062 & 0.764 & 0.977 & 1.164 & 0.467 & - & - & - & - & - \\
\hline 0.275 & 0.770 & 0.821 & 1.123 & 0.871 & 1.094 & 1.292 & 0.496 & - & - & - & - & - \\
\hline 0.300 & 0.759 & 0.813 & 1.122 & 0.803 & 0.996 & 1.158 & 0.559 & -0.513 & - & 0.215 & - & - \\
\hline 0.325 & 0.806 & 0.854 & 1.143 & 0.772 & 0.971 & 1.146 & 0.587 & -0.235 & - & 0.673 & - & - \\
\hline 0.350 & 0.812 & 0.858 & 1.143 & 0.735 & 0.937 & 1.121 & 0.664 & -0.077 & - & 0.886 & - & - \\
\hline 0.375 & 0.816 & 0.860 & 1.140 & 0.699 & 0.900 & 1.090 & 0.733 & 0.042 & - & 1.082 & - & - \\
\hline 0.400 & 0.818 & 0.860 & 1.135 & 0.662 & 0.861 & 1.056 & 0.798 & 0.144 & - & 1.208 & - & - \\
\hline 0.425 & 0.817 & 0.858 & 1.127 & 0.626 & 0.821 & 1.018 & 0.860 & 0.236 & - & 1.318 & - & - \\
\hline 0.450 & 0.814 & 0.854 & 1.118 & 0.589 & 0.780 & 0.978 & 0.918 & 0.318 & - & 1.417 & - & - \\
\hline 0.475 & 0.809 & 0.848 & 1.107 & 0.553 & 0.738 & 0.936 & 0.973 & 0.390 & - & 1.509 & - & - \\
\hline 0.500 & 0.803 & 0.841 & 1.094 & 0.516 & 0.696 & 0.891 & 1.027 & 0.454 & - & 1.597 & - & - \\
\hline 0.525 & 0.795 & 0.832 & 1.080 & 0.480 & 0.652 & 0.845 & 1.079 & 0.510 & - & 1.681 & - & - \\
\hline 0.550 & 0.787 & 0.822 & 1.064 & 0.444 & 0.608 & 0.797 & 1.130 & 0.506 & - & 1.764 & - & - \\
\hline 0.575 & 0.777 & 0.811 & 1.047 & 0.408 & 0.563 & 0.748 & 1.179 & 0.606 & - & 1.846 & - & - \\
\hline 0.600 & 0.765 & 0.798 & 1.028 & 0.372 & 0.518 & 0.697 & 1.227 & 0.648 & - & 1.925 & - & - \\
\hline 0.625 & 0.753 & 0.784 & 1.007 & 0.336 & 0.472 & 0.645 & 1.275 & 0.687 & - & 2.003 & - & - \\
\hline 0.650 & 0.729 & 0.760 & 0.979 & 0.301 & 0.427 & 0.591 & 1.320 & 0.723 & -1.405 & 2.079 & -1.646 & -1.392 \\
\hline 0.675 & 0.707 & 0.737 & 0.951 & 0.264 & 0.381 & 0.538 & 1.362 & 0.754 & -0.551 & 2.153 & -0.797 & -0.563 \\
\hline 0.700 & 0.685 & 0.714 & 0.922 & 0.227 & 0.335 & 0.484 & 1.402 & 0.784 & -0.161 & 2.227 & -0.400 & -0.202 \\
\hline 0.725 & 0.660 & 0.688 & 0.889 & 0.190 & 0.288 & 0.428 & 1.442 & 0.812 & 0.115 & 2.300 & -0.114 & 0.045 \\
\hline 0.750 & 0.634 & 0.660 & 0.854 & 0.151 & 0.240 & 0.371 & 1.480 & 0.841 & 0.332 & 2.375 & 0.112 & 0.237 \\
\hline 0.775 & 0.605 & 0.630 & 0.816 & 0.111 & 0.191 & 0.312 & 1.518 & 0.868 & 0.520 & 2.447 & 0.305 & 0.394 \\
\hline 0.800 & 0.574 & 0.598 & 0.781 & 0.070 & 0.140 & 0.251 & 1.555 & 0.895 & 0.684 & 2.523 & 0.472 & 0.526 \\
\hline 0.825 & 0.528 & 0.552 & 0.739 & 0.020 & 0.083 & 0.184 & 1.591 & 0.921 & 0.887 & 2.629 & 0.675 & 0.654 \\
\hline 0.850 & 0.505 & 0.529 & 0.710 & -0.017 & 0.035 & 0.124 & 1.627 & 0.948 & 0.979 & 2.686 & 0.770 & 0.745 \\
\hline 0.875 & 0.469 & 0.492 & 0.672 & -0.062 & -0.018 & 0.059 & 1.662 & 0.974 & 1.110 & 2.773 & 0.888 & 0.828 \\
\hline 0.900 & 0.434 & 0.457 & 0.633 & -0.105 & -0.069 & -0.004 & 1.696 & 1.001 & 1.221 & 2.857 & 0.993 & 0.908 \\
\hline 0.925 & 0.410 & 0.432 & 0.600 & -0.142 & -0.113 & -0.061 & 1.730 & 1.027 & 1.307 & 2.925 & 1.054 & 0.949 \\
\hline 0.950 & 0.374 & 0.395 & 0.557 & -0.184 & -0.162 & -0.121 & 1.762 & 1.053 & 1.416 & 3.019 & 1.135 & 0.997 \\
\hline 0.975 & 0.352 & 0.372 & 0.523 & -0.215 & -0.199 & -0.169 & 1.794 & 1.080 & 1.485 & 3.097 & 1.179 & 1.032 \\
\hline 1.000 & 0.311 & 0.330 & 0.473 & -0.257 & -0.246 & -0.224 & 1.824 & 1.106 & 1.603 & 3.213 & 1.244 & 1.060 \\
\hline
\end{tabular}

\section{Note}

1 Neutrons and $\Lambda$ hyperons also couple to the electromagnetic fields due to their magnetic moments; however, this contribution is negligible.

\section{References}

1. Blaschke, D.; Chamel, N. Phases of Dense Matter in Compact Stars. In Astrophysics and Space Science Library; Rezzolla, L., Pizzochero, P., Jones, D.I., Rea, N., Vidaña, I., Eds.; Springer: Cham, Switzerland , 2018; Volume 457, p. 337. [CrossRef]

2. Ambartsumyan, V.A.; Sakyan, G.S. The Degenerate Superdense Gas of Elementary Particles. Sov. Astron. $1960,4,187$.

3. Balberg, S.; Gal, A. An effective equation of state for dense matter with strangeness. Nucl. Phys. A 1997, 625, 535. [CrossRef]

4. Balberg, S.; Lichtenstadt, I.; Cook, G.B. Roles of hyperons in neutron stars. Astrophys. J. Suppl. Ser. 1999, 121, 515. [CrossRef] 
5. Glendenning, N.K. The hyperon composition of neutron stars. Phys. Lett. B 1982, 114, 392. [CrossRef]

6. Glendenning, N.K. Neutron stars are giant hypernuclei? Astrophys. J. 1985, 293, 470. [CrossRef]

7. Glendenning, N.K. Hyperons in neutron stars. Z. Phys. A 1987, 326, 57. [CrossRef]

8. Glendenning, N.K.; Moszkowski, S.A. Reconciliation of neutron-star masses and binding of the $\Lambda$ hypernuclei. Phys. Rev. Lett. 1991, 67, 2414. [CrossRef]

9. Weber, F.; Weigel, M.K. Baryon Composition and Macroscopic Properties of Neutron Stars. Nucl. Phys. A 1989, 505, 779. [CrossRef]

10. Knorren, R.; Prakash, M.; Ellis, P.J. Strangeness in hadronic stellar matter. Phys. Rev. C 1995, 52, 3470. [CrossRef]

11. Schaffner, J.; Mishustin, I. Hyperon-rich matter in neutron stars. Phys. Rev. C 1996, 53, 1416. [CrossRef] [PubMed]

12. Huber, H.; Weber, F.; Weigel, M.K.; Schaab, C. Neutron star properties with relativistic equations of state. Int. J. Mod. Phys. E 1998, 7, 310. [CrossRef]

13. Schulze, H.J.; Baldo, M.; Lombardo, U.; Cugnon, J.; Lejeune, A. Hypernuclear matter in the Brueckner-Hartree-Fock approximation. Phys. Lett. B 1995, 355, 21. [CrossRef]

14. Schulze, H.J.; Baldo, M.; Lombardo, U.; Cugnon, J.; Lejeune, A. Hyperonic nuclear matter in Brueckner theory. Phys. Rev. C 1998, 57, 704. [CrossRef]

15. Baldo, M.; Burgio, G.F.; Schulze, H.J. Onset of hyperon formation in neutron star matter from Brueckner theory. Phys. Rev. C 1998, 58, 3688. [CrossRef]

16. Vidaña, I.; Polls, A.; Ramos, A.; Hjorth-Jensen, M.; Stoks, V.G.J. Strange nuclear matter within Brueckner-Hartree-Fock theory. Phys. Rev. C 2000, 61, 025802. [CrossRef]

17. Baldo, M.; Burgio, G.F.; Schulze, H.J. Hyperon stars in the Brueckner-Bethe-Goldstone theory. Phys. Rev. C 1998, 61, 055801. [CrossRef]

18. Vidaña, I.; Polls, A.; Ramos, A.; Engvik, L.; Hjorth-Jensen, M. Hyperon-hyperon interactions and properties of neutron star matter. Phys. Rev. C 2000, 62, 035801. [CrossRef]

19. Schulze, H.J.; Polls, A.; Ramos, A.; Vidaña, I. Maximum mass of neutron stars. Phys. Rev. C 2006, 73, 058801. [CrossRef]

20. Sammarruca, F. Effect of $\Lambda$ hyperons on the nuclear equation of state in a Dirac-Brueckner-Hartree-Fock model. Phys. Rev. C 2009, 79, 034301. [CrossRef]

21. Dapo, H.; Schaeffer, B.J.; Wambach, J. Appearance of hyperons in neutron stars. Phys. Rev. C 2010, 81, 035803. [CrossRef]

22. Schulze, H.J.; Rijken, T. Maximum mass of hyperon stars with the Nijmegen ESC08 model. Phys. Rev. C 2011, 84, 035801. [CrossRef]

23. Lonardoni, D.; Pederiva, F.; Gandolfi, S. Accurate determination of the interaction between $\Lambda$ hyperons and nucleons from auxiliary field diffusion Monte Carlo calculations. Phys. Rev. C 2014, 89, 014314. [CrossRef]

24. Lonardoni, D.; Lovato, A.; Gandolfi, S.; Pederiva, F. Hyperon Puzzle: Hints from Quantum Monte Carlo Calculations. Phys. Rev. Lett. 2015, 114, 092301. [CrossRef]

25. Petschauer, S.; Haidenbauer, J.; Kaiser, N.; Meissner, U.G.; Weise, W. Hyperons in nuclear matter from SU(3) chiral effective field theory. Eur. Phys. J. A 2016, 52, 15. [CrossRef]

26. Logoteta, D.; Vidaña, I.; Bombaci, I. Impact of chiral hyperonic three-body forces on neutron stars. Eur. Phys. J. A 2019, 57, 207. [CrossRef]

27. Champion, D.J.; Ransom, S.M.; Lazarus, P.; Camilo, F.; Bassa, C.; Kaspi, V.M.; Nice, D.J.; Freire, P.C.C.; Stairs, I.H.; van Leeuwen, J.; et al. An eccentric binary millisecond pulsar in the galactic plane. Science 2008, 320, 1309. [CrossRef]

28. Demorest, P.B.; Pennucci, T.; Ransom, S.M.; Roberts, M.S.E.; Hessels, J.W.T. A two-solar-mass neutron star measured using Shapiro delay. Nature 2010, 467, 1081. [CrossRef]

29. Antoniadis, J.; Freire, P.C.C.; Wex, N.; Tauris, T.M.; Lynch, R.S.; van Kerkwijk, M.H.; Kramer, M.; Bassa, C.; Dhillon, V.S.; Driebe, T.; et al. A massive pulsar in a compact relativistic binary. Science 2013, 340, 1233232. [CrossRef]

30. Cromartie, H.T.; Fonseca, E.; Ransom, S.M.; Demorest, P.B.; Arzoumanian, Z.; Blumer, H.; Brook, P.R.; DeCesar, M.E.; Dolch, T.; Ellis, J.A.; et al. Relativistic Shapiro delay measurements of an extremely massive millisecond pulsar. Nat. Astron. $2020,4,72$. [CrossRef]

31. Chatterjee, D.; Vidaña, I. Do hyperons exists in the interior of neutron stars? Eur. Phys. J. A 2016, 52, 29. [CrossRef]

32. Vidaña, I. Hyperons: the strange ingredients of the nuclear equation of state. Proc. R. Soc. Lond. Ser. A 2018, $474,20180145$. [CrossRef]

33. Kaplan, D.B.; Nelson, A.E. Strange goings on in dense nucleonic matter. Phys. Lett. B 1986, 175, 57-63; Erratum in 1986, 179, 409-410. [CrossRef]

34. Brown, G.E.; Lee, C.H.; Rho, M.; Thorsson, V. From kaon-nuclear interactions to kaon condensation. Nucl. Phys. A 1994, 567, 937-956. [CrossRef]

35. Thorsson, V.; Prakash, M.; Lattimer, J.M. Composition, structure and evolution of neutron stars with kaon condensates. Nucl. Phys. A 1994, 572, 693-731. [CrossRef]

36. Lee, C.H. Kaon condensation in dense stellar matter. Phys. Rep. 1996, 275, 255-341. [CrossRef]

37. Glendenning, N.K.; Schaffner-Bielich, J. Kaon Condensation and Dynamical Nucleons in Neutron Stars. Phys. Rev. Lett. 1998, 81, 4564-4567. [CrossRef]

38. Tolos, L.; Fabbietti, L. Strangeness in nuclei and neutron stars. Prog. Part. Nucl. Phys. 2020, 112, 103770. [CrossRef] 
39. Schmitt, A.; Shternin, P. Reaction Rates and Transport in Neutron Stars. In The Physics and Astrophysics of Neutron Stars; Rezzolla, L., Pizzochero, P., Jones, D.I., Rea, N., Vidaña, I., Eds.; Springer International Publishing: Cham, Switzerland, 2018; pp. 455-574. [CrossRef]

40. Yakovlev, D.G.; Shalybkov, D.A. Electrical conductivity of neutron star cores in the presence of a magnetic field-I. General solution for a multicomponent Fermi liquid-II.-A free particle model of npe $\Sigma^{-}$matter. Astroph. Space Sci. 1991, 176, 171-189. [CrossRef]

41. Goldreich, P.; Reisenegger, A. Magnetic field decay in isolated neutron stars. Astrophys. J. 1992, 395, 250-258. [CrossRef]

42. Dommes, V.A.; Gusakov, M.E.; Shternin, P.S. Dissipative relativistic magnetohydrodynamics of a multicomponent mixture and its application to neutron stars. Phys. Rev. D 2020, 101, 103020. [CrossRef]

43. Baym, G.; Pethick, C. Landau Fermi-Liquid Theory: Concepts and Applications; John Wiley \& Sons, Inc.: New York, NY, USA; Chichester, UK; Brisbane, Australia; Toronto, ON, Canada; Singapore, 1991; [CrossRef]

44. Flowers, E.; Itoh, N. Transport properties of dense matter. II. Astrophys. J. 1979, 230, 847-858. [CrossRef]

45. Anderson, R.H.; Pethick, C.J.; Quader, K.F. Transport properties of a multicomponent Fermi liquid. Phys. Rev. B 1987, 35, 1620-1629. [CrossRef]

46. Shternin, P.S.; Baldo, M.; Haensel, P. Transport coefficients of nuclear matter in neutron star cores. Phys. Rev. C 2013, 88, 065803. [CrossRef]

47. Shternin, P.S.; Baldo, M. Transport coefficients of nucleon neutron star cores for various nuclear interactions within the BruecknerHartree-Fock approach. Phys. Rev. D 2020, 102, 063010. [CrossRef]

48. Baldo, M. (Ed.) Nuclear Methods and the Nuclear Equation of State; Volume 8, International Review of Nuclear Physics; World Scientific: Singapore, 1999; [CrossRef]

49. Wiringa, R.B.; Stoks, V.G.J.; Schiavilla, R. Accurate nucleon-nucleon potential with charge-independence breaking. Phys. Rev. C 1995, 51, 38-51. [CrossRef] [PubMed]

50. Carlson, J.; Pandharipande, V.R.; Wiringa, R.B. Three-nucleon interaction in 3-, 4- and N-body systems. Nucl. Phys. A 1983, 401, 59-85. [CrossRef]

51. Stoks, V.G.J.; Rijken, T.A. Soft-core baryon-baryon potentials for the complete baryon octet. Phys. Rev. C 1999, 59, 3009-3020. [CrossRef]

52. Rijken, T.A.; Stoks, V.G.J.; Yamamoto, Y. Soft-core hyperon-nucleon potentials. Phys. Rev. C 1999, 59, 21-40. [CrossRef]

53. Shternin, P.; Baldo, M.; Schulze, H. Transport coefficients in neutron star cores in BHF approach. Comparison of different nucleon potentials. J. Phys. Conf. Ser. 2017, 932, 012042. [CrossRef]

54. Shternin, P. Transport coefficients of leptons in superconducting neutron star cores. Phys. Rev. D 2018, 98, 063015. [CrossRef]

55. Heiselberg, H.; Pethick, C.J. Transport and relaxation in degenerate quark plasmas. Phys. Rev. D 1993, 48, 2916-2928. [CrossRef] [PubMed]

56. Heiselberg, H.; Baym, G.; Pethick, C.J.; Popp, J. Transport coefficients of relativistic plasmas. Nucl. Phys. A 1992, 544, 569-572. [CrossRef]

57. Akmal, A.; Pandharipande, V.R.; Ravenhall, D.G. Equation of state of nucleon matter and neutron star structure. Phys. Rev. C 1998, 58, 1804-1828. [CrossRef]

58. Fabrocini, F.; Fantoni, S. Correlated basis function results for the Argonne models of nuclear matter. Phys. Lett. B 1993, 298, 263-266. [CrossRef]

59. Kadanoff, L.P.; Baym, G. Quantum Statistical Mechanics; Benjamin: New York, NY, USA, 1962.

60. Kraeft, W.D.; Kremp, W.; Ebeling, W.; Röpke, G. Quantum Statistical of Charged Paerticle Systems; Akademie-Verlag: Berlin, Germany, 1986.

61. Day, B.D. Elements of the Brueckner-Goldstone Theory of Nuclear Matter. Rev. Mod. Phys. 1967, 39, 719-744. [CrossRef]

62. ter Haar, B.; Malfliet, R. Nucleons, mesons and deltas in nuclear matter a relativistic Dirac-Brueckner approach. Phys. Rep. 1987, 149, 207-286. [CrossRef]

63. ter Haar, B.; Malfliet, R. Pion production, pion absorption, and nucleon properties in dense nuclear matter: Relativistic Dirac-Brueckner approach at intermediate and high energies. Phys. Rev. C 1987, 36, 1611-1620. [CrossRef] [PubMed]

64. Brockman, R.; Machleidt, R. Relativistic nuclear structure. I. Nuclear matter. Phys. Rev. C 1990, 42, 1965-1980. [CrossRef]

65. Song, H.Q.; Baldo, M.; Giansiracura, G.; Lombardo, U. Bethe-Brueckner-Goldstone expansion in nuclear matter. Phys. Rev. Lett. 1998, 81, 1584. [CrossRef]

66. Baldo, M.; Giansiracusa, G.; Lombardo, U.; Song, H.Q. Bethe-Brueckner-Goldstone expansion in neutron matter. Phys. Lett. B 2000, 473, 1. [CrossRef]

67. Loiseau, A.; Nogami, Y.; Ross, C.K. Nucleon-nucleon correlation and two-pion-exchange three-body force in nuclear matter. Nucl. Phys. A 1971, 165, 601. [CrossRef]

68. Grangé, P.; Martzolff, M.; Nogami, Y.; Sprung, D.W.L.; Ross, C.K. Three-body force in nuclear matter. Phys. Lett. B 1976, 60, 237. [CrossRef]

69. Baldo, M.; Ferreira, L. Nuclear liquid-gas phase transition. Phys. Rev. C 1999, 59, 682. [CrossRef]

70. Baldo, M.; Burgio, G.F.; Schulze, H.J.; Taranto, G. Nucleon effective masses within the Brueckner-Hartree-Fock theory: Impact on stellar neutrino emission. Phys. Rev. C 2014, 89, 048801. [CrossRef] 
71. Shternin, P.S.; Yakovlev, D.G. Electron-muon heat conduction in neutron star cores via the exchange of transverse plasmons. Phys. Rev. D 2007, 75, 103004. [CrossRef]

72. Shternin, P.S.; Yakovlev, D.G. Shear viscosity in neutron star cores. Phys. Rev. D 2008, 78, 063006. [CrossRef]

73. Shternin, P.S. Exchange of transverse plasmons and electrical conductivity of neutron star cores. JETP 2008, 107, 212-227. [CrossRef]

74. Sedrakian, A.; Clark, J.W. Superfluidity in nuclear systems and neutron stars. Eur. Phys. J. A 2019, 55, 167. [CrossRef]

75. Balberg, S.; Barnea, N. S-wave pairing of $\Lambda$ hyperons in dense matter. Phys. Rev. C 1998, 57, 409-416. [CrossRef]

76. Takatsuka, T.; Tamagaki, R. Superfluidity of $\Lambda$ hyperons admixed in neutron star cores. Prog. Theor. Phys. 1999, 102, 1043-1048. [CrossRef]

77. Takatsuka, T.; Nishizaki, S.; Yamamoto, Y.; Tamagaki, R. Possibility of hyperon superfluidity in neutron star cores. Prog. Theor. Phys. 2000, 107, 179-184. [CrossRef]

78. Takatsuka, T.; Nishizaki, S.; Yamamoto, Y.; Tamagaki, R. Superfluidity of hyperon-mixed neutron stars. Prog. Theor. Phys. Suppl. 2002, 146, 279-288. [CrossRef]

79. Vidaña, I.; Tolós, L. Superfluidity of $\Sigma^{-}$hyperons in $\beta$-stable neutron star matter. Phys. Rev. C 2004, 70, 02882. [CrossRef]

80. Zhou, X.R.; Schulze, H.J.; Pan, E.; Drayer, J.P. Strong hyperon-nucleon pairing in neutron stars. Phys. Rev. Lett. 2005, 95, 051101. [CrossRef] [PubMed]

81. Wang, Y.N.; Shen, H. Superfluidity of $\Lambda$ hyperons in neutron stars. Phys. Rev. C 2010, 81, 025801. [CrossRef]

82. Andersson, N. A Superfluid Perspective on Neutron Star Dynamics. Universe 2021, 7, 17. [CrossRef]

83. Baiko, D.A.; Haensel, P.; Yakovlev, D.G. Thermal conductivity of neutrons in neutron star cores. Astron. Astrophys. 2001, 374, 151-163. [CrossRef]

84. Vollhardt, D.; Wölfle, P. The Superfluid Phases of Helium 3; Taylor \& Francis: Bristol, UK, 1990; [CrossRef]

85. Manuel, C.; Tolos, L. Transport Properties of Superfluid Phonons in Neutron Stars. Universe 2021, 7, 59. [CrossRef] 